\title{
Numerical Green's Functions for Line Force and Dislocation around Multiple Cracks
}

\author{
M. Denda and P. Quick \\ Rutgers University, Mech. \& Aero. Engng. Dept. \\ Piscataway, NJ 08854-8058, U.S.A.
}

\begin{abstract}
The numerical Green's function technique for an infinite isotropic domain with multiple cracks is developed. The singularities considered are the line force and dislocation. The Green's function is decomposed into the singular and the image terms. To obtain the image term we represent the crack opening displacement (COD) by the dislocation dipole distribution, embed the $\sqrt{r}$ crack tip behavior, and integrate the resulting singular/hyper-singular integrals analytically. The resulting whole crack singular element (WCSE) consists of multiple independent crack opening modes and is strictly algebraic with the correct crack tip singular behavior but the magnitude for each mode is unknown. They are determined to give the negative of the crack surface traction induced by the singular term. Extensive error analysis is performed for the line force and dislocation in an infinite domain with a single crack to identify the region where, when these singularities are placed, the solution achieves high accuracy. Following the guideline set by the error analysis, numerical Green's functions for a few multiple crack configurations are obtained for the line force and dislocation.
\end{abstract}

\section{Introduction}

The fundamental solutions, such as for the line force and dislocation, are defined in an infinite homogeneous body and their main characteristic is the singularity. When defects (such as cracks and holes) and inhomogeneities (such as inclusions) are introduced the fundamental solutions do not satisfy their required boundary conditions for the defects any more and additional terms are needed if these boundary conditions are enforced. The fundamental solution augmented by the additional image term that satisfy the required boundary conditions is called the Green's function. The fundamental solutions considered in this paper are for the line force and dislocation. These fundamental solutions serve as the essential building block for the solutions of the linear elastic problems by the boundary element method (BEM). Given these fundamental solutions, we establish a technique to determine their Green's functions numerically when multiple cracks are present in two-dimensional isotropic solids. Such Green's functions are called the numerical Green's functions by Telles et al. [1, 2, 3]

The majority of the Green's functions are analytical and are concerned about the simplest defect/inhomogeneity geometries possible such as the single center crack(Snider and Cruse [4], Cruse [5], Clements and Haselgrove [6]) or interface crack(Berger and Tewary [7], Yuuki and Cho [8]), the single elliptical hole (Morjaria and Mukherjee [9], Ang and Clements [10], Kamel and Liaw [11], Hwu and 
Yen [12], Denda and Kosaka [13]), and the half-plane/bimaterial domain (Telles and Brebbia [14], Meek and Dai [15], Dumir and Mehta [16], Pan et al. [17], Berger [18], Denda [19]). The only simple geometric configurations have allowed the analytical derivation of these Green's functions. The numerical Green's function technique of Telles $[1,2,3]$, aimed for crack problems, has broken this limitation and enabled the derivation of the Green's functions for more complex twodimensional and three-dimensional crack configurations. The Green's function is decomposed into the singular and the image terms, the latter being the solution with the negative of the traction loading on the crack surfaces induced by the former. The image term is expressed as the function of the unknown crack opening displacement (COD) which are determined to satisfy, when the singular solution is superposed, the traction free condition on the crack surfaces. Telles et al. [1] have set up the hyper-singular integral equations for the crack surface loading of the image term and evaluated the integral by the numerical quadrature to determine the CODs. In this paper we propose a technique to evaluate the singular and hyper-singular integrals analytically using the complex variable theory. We model the crack opening displacement by the dislocation dipole distribution, which is interpolated by the product of $\sqrt{a^{2}-x^{2}}$ and the orthogonal polynomials, where $a$ is the half-crack length of a straight crack along the $x$-axis. The resulting Cauchytype integrals are evaluated analytically to set up a departure from the quadrature formula approach of Telles. Even though the singular and hyper-singular integrals are evaluated analytically, the CODs still have to be determined numerically, thus the technique is called the numerical Green's function.

When the singularity approaches the prospective crack, its traction distribution along the prospective crack has a sharp peak at the point closest to the singularity. The $p$-type COD interpolation of the WCSE cannot take this behavior well. This sets the limitation of the numerical Green's function. We perform an extensive error analysis of the numerical Green's functions for the single crack, for which the analytical Green's functions are available (Denda and Kosaka [13]). We will identify the zone of applicability of the proposed numerical Green's functions where they can be confidently applied.

\section{Fundamental solutions in elasticity}

\subsection{Line force and dislocation}

The fundamental (or singularity) solutions of elasticity consist of the line force and dislocation. We use Muskhelishvili's [20] complex variable formalism, as summarized in Appendix A.1, for linear isotropic elasticity to derive them. Consider a line force $f=f_{x}+i f_{y}$ or dislocation $b=b_{x}+i b_{y}$ at $\xi$ in the infinite plane, where $b$ is Burgers vector of the dislocation. The displacement at $z=x+i y$, using the results in Appendix A.2, is given by

$$
u^{(s)}(z, \xi) \equiv u_{x}^{(s)}+i u_{y}^{(s)}=-\frac{\gamma}{2 \mu}\{\kappa \log (z-\xi)-k \overline{\log (z-\xi)}\}+\frac{\bar{\gamma}}{2 \mu} \frac{(z-\xi)}{(\overline{z-\xi})},
$$


and the stress components by

$$
\begin{gathered}
\sigma^{(s)}(z, \xi) \equiv \frac{\sigma_{x x}^{(s)}+\sigma_{y y}^{(s)}}{2}=-2 \operatorname{Re}\left[\frac{\gamma}{(z-\xi)}\right], \\
\tau^{(s)}(z, \xi) \equiv \frac{\sigma_{y y}^{(s)}-\sigma_{x x}^{(s)}}{2}+i \sigma_{x y}^{(s)}=\gamma \frac{(\overline{z-\xi})}{(z-\xi)^{2}}-\bar{\gamma} \frac{k}{(z-\xi)},
\end{gathered}
$$

where

$$
\left\{\begin{array}{lll}
k=-\kappa, & \gamma=f / 2 \pi(\kappa+1) & \text { line force } \\
k=1, & \gamma=i \mu b / \pi(\kappa+1) & \text { line dislocation, }
\end{array}\right.
$$

$\mu$ is the shear modulus and $\kappa$ is Muskhelishvili constant defined in Appendix A.1. The traction $t=t_{x}+i t_{y}$ at $z$ on a line segment with the unit normal $n_{x}+i n_{y}=e^{i \alpha}$ is given by

$t^{(s)}(z, \alpha ; \xi)=-\gamma\left\{e^{i \alpha} \frac{1}{(z-\xi)}-e^{-i \alpha} \frac{k}{\overline{(z-\xi)}}\right\}-\bar{\gamma}\left\{e^{i \alpha} \frac{1}{(\overline{z-\xi})}+e^{-i \alpha} \frac{(z-\xi)}{(\overline{z-\xi})^{2}}\right\}$.

\section{Whole crack singular element}

Consider a dislocation dipole, which is constructed by a pair of line dislocations with opposite Burgers vectors $-b$ and $b$ located at $\xi$ and $\xi+d \xi$, respectively. It represents the displacement jump along the infinitesimal segment $d \xi$ at $\xi$. The displacement and stress components, obtained by taking the total derivative of the line dislocation solutions (1) and (2) with respect to $\xi$, are given by

$$
d u^{(s)}(z, \xi)=\frac{i}{2 \pi(\kappa+1)}\left\{b\left[\frac{\kappa d \xi}{(z-\xi)}-\frac{d \bar{\xi}}{(\overline{z-\xi})}\right]+\bar{b}\left[\frac{d \xi}{(\overline{z-\xi})}-\frac{(z-\xi)}{(\overline{z-\xi})^{2}} d \bar{\xi}\right]\right\}
$$

and

$$
\begin{aligned}
d \sigma^{(s)}(z, \xi) & =-2 \operatorname{Re}\left[\frac{i \mu b}{\pi(\kappa+1)} \frac{d \xi}{(z-\xi)^{2}}\right] \\
d \tau^{(s)}(z, \xi) & =\frac{i \mu}{\pi(\kappa+1)}\left\{b\left[-\frac{d \bar{\xi}}{(z-\xi)^{2}}+2 \frac{(\overline{z-\xi})}{(z-\xi)^{3}} d \xi\right]+\bar{b} \frac{d \xi}{(z-\xi)^{2}}\right\} .
\end{aligned}
$$

The traction is given by

$$
\begin{aligned}
d t^{(s)}(z, \xi)=-\frac{i \mu e^{-i \alpha}}{\pi(\kappa+1)}\left\{b\left[\frac{e^{i 2 \alpha}}{(z-\xi)^{2}} d \xi-\frac{1}{(\overline{z-\xi})^{2}} d \bar{\xi}\right]\right. \\
\left.-\bar{b}\left[\frac{e^{i 2 \alpha}}{(\overline{z-\xi})^{2}} d \bar{\xi}-\frac{1}{(\overline{z-\xi})^{2}} d \xi+2 \frac{(z-\xi)}{(\overline{z-\xi})^{3}} d \bar{\xi}\right]\right\}
\end{aligned}
$$


Consider the straight center crack in the infinite body. We represent the crack opening displacement by the dislocation dipole distribution along the crack and call it the crack element. Denda and Dong [21] has interpolated the $\sqrt{r}$ COD behavior at the crack tip and integrated the dislocation dipole distribution analytically to develop the whole crack singular element (WCSE). We will recapitulate the key formulas for the WCSE below. Introduce a local coordinate system $O-x y$ with the coordinate origin $O$ at the crack center and the $x$-axis along the crack so that the crack lies in the interval $(-a, a)$. The local complex variable is denoted by $z=x+i y$ and the crack opening displacement by $\delta=\delta_{x}+i \delta_{y}$. If we normalize the local coordinates by $Z=z / a$ (i.e., $X=x / a$ and $Y=y / a$ ), then the crack interval is reduced to $(-1,+1)$ and the complex potential functions of the crack element are given, from (A.9) with $k=1$, by

$$
\begin{aligned}
\phi^{(s)}(Z) & =\int_{-1}^{+1} \tilde{\gamma}(X) \frac{d X}{Z-X}, \\
\psi^{(s)}(Z) & =\int_{-1}^{+1}\{\tilde{\gamma}(X)+\overline{\tilde{\gamma}(X)}\} \frac{d X}{Z-X}-\frac{\partial}{\partial Z}\left\{\int_{-1}^{+1} \tilde{\gamma}(X) \frac{X d X}{Z-X}\right\},
\end{aligned}
$$

where

$$
\tilde{\gamma}(X)=\gamma(x)=i \mu \delta / \pi(\kappa+1) .
$$

Interpolate the density function $\tilde{\gamma}(X)$ by

$$
\tilde{\gamma}(X)=\frac{i \mu}{\pi(\kappa+1)} \sqrt{1-X^{2}} \sum_{m=1}^{M} \delta^{(m)} U_{m-1}(X),
$$

which embed the $\sqrt{r}$ COD behavior at the crack tips. The unknown crack opening displacement coefficients $\delta^{(m)}$ are determined following the procedure described later. Use (C.2) to integrate (8), with the density function (9), analytically with the result

$$
\begin{aligned}
\phi^{(s)}(Z) & =\frac{i \mu}{(\kappa+1)} \sum_{m=1}^{M} \delta^{(m)} T^{(m)}(Z) \\
\psi^{(s)}(Z) & =-\frac{i \mu}{(\kappa+1)} \sum_{m=1}^{M}\left\{\overline{\delta^{(m)}} T^{(m)}(Z)+m \delta^{(m)} Z U^{(m-1)}(Z)\right\}
\end{aligned}
$$

where $T^{(m)}(Z)$ and $U^{(m-1)}(Z)$ are given by (C.2) of Appendix C. The displacement contribution is given, from (10) and (A.1), by

$$
u=\sum_{m=1}^{M}\left(K^{(m)}(Z) \delta^{(m)}+\overline{L^{(m)}(Z) \delta^{(m)}}\right),
$$

where

$$
\begin{aligned}
K^{(m)}(Z) & =\frac{i}{2(\kappa+1)}\left\{\kappa T^{(m)}(Z)-\overline{T^{(m)}(Z)}\right\} \\
L^{(m)}(Z) & =\frac{i}{2(\kappa+1)}(Z-\bar{Z}) m U^{(m-1)}(Z)
\end{aligned}
$$


and the stress contribution, from (10) and (A.2), by

$$
\begin{aligned}
\sigma & =\sum_{m=1}^{M}\left(P^{(m)}(Z) \delta^{(m)}+\overline{Q^{(m)}(Z) \delta^{(m)}}\right), \\
\tau & =\sum_{m=1}^{M}\left(R^{(m)}(Z) \delta^{(m)}+\overline{S^{(m)}(Z) \delta^{(m)}}\right),
\end{aligned}
$$

where

$$
\begin{aligned}
P^{(m)}(Z) & =Q^{(m)}(Z)=\frac{i}{a(\kappa+1)} m U^{(m-1)}(Z), \\
R^{(m)}(Z) & =-\frac{i}{a(\kappa+1)} m\left[U^{(m-1)}(Z)+\frac{Z-\bar{Z}}{Z^{2}-1}\left\{(m+1) T^{(m)}(Z)-U^{(m)}(Z)\right\}\right], \\
S^{(m)}(Z) & =\frac{i}{a(\kappa+1)} m \overline{U^{(m-1)}(Z)} .
\end{aligned}
$$

The traction on the line segment whose unit normal has slope $\alpha$ is given, from (13) and (A.3), by

$$
t=\sum_{m=1}^{M}\left\{K^{*(m)}(Z, \alpha) \delta^{(m)}+\overline{L^{*(m)}(Z, \alpha) \delta^{(m)}}\right\}
$$

where

$$
\begin{aligned}
K^{*(m)}(Z, \alpha) & =\frac{i \mu m}{a(\kappa+1)}\left\{e^{i \alpha} U^{(m-1)}(Z)-e^{-i \alpha} \overline{U^{(m-1)}(Z)}\right\}, \\
L^{*(m)}(Z, \alpha) & =\frac{i \mu m}{a(\kappa+1)}\left\{\left(e^{i \alpha}+e^{-i \alpha}\right) U^{(m-1)}(Z)\right. \\
& \left.+e^{i \alpha} \frac{Z-\bar{Z}}{Z^{2}-1}\left[(m+1) T^{(m)}(Z)-U^{(m)}(Z)\right]\right\} .
\end{aligned}
$$

Evaluate (15) along the crack line to get the traction

$$
t^{+}=\frac{2 \mu}{a(\kappa+1)} \sum_{m=1}^{M} m \delta^{(m)} U_{m-1}(X) \quad(|X| \leq 1),
$$

on the upper crack surface and the stress on the $X$-axis outside the crack

$$
\sigma_{y y}+i \sigma_{x y}= \pm \frac{2 \mu i}{a(\kappa+1)} \sum_{m=1}^{M} m \overline{\delta^{(m)}} \frac{\left(X \mp{\sqrt{X^{2}-1}}^{m}\right.}{\sqrt{X^{2}-1}},
$$

where the upper and lower signs correspond to $X>1$ and $X<-1$, respectively. The stress intensity factor is extracted, from (18), as

$$
K( \pm 1)=K_{I}( \pm 1)+i K_{I I}( \pm 1)=\frac{2 \mu i}{\kappa+1} \sqrt{\frac{\pi}{a}} \sum_{m=1}^{M}( \pm 1)^{m+1} m \overline{\delta^{(m)}},
$$

where $K_{I}$ and $K_{I I}$ are the Mode I and II stress intensity factors in the local $O$-xy coordinate system. 


\section{Numerical Green's functions}

\subsection{Single horizontal crack}

Consider an infinite body with a straight crack in the interval $(-a, a)$ along the $x$-axis and apply either the line force or dislocation to the cracked body.

\subsubsection{Determination of crack opening displacement coefficients}

Let $\phi^{(s)}(z)$ and $\psi^{(s)}(z)$ be the complex potential functions for the fundamental solution. The traction $t^{(s)}=t_{x}^{(s)}+i t_{y}^{(s)}$ on the crack surface due to the fundamental solutionis does not, in general, vanish. To satisfy the traction free condition we seek the solution in the form

$$
\phi^{(t o t a l)}(z)=\phi^{(s)}(z)+\phi(z), \quad \psi^{(t o t a l)}(z)=\psi^{(s)}(z)+\psi(z),
$$

where the image terms $\phi(z)$ and $\psi(z)$ are required to produce the traction $t=$ $t_{x}+i t_{y}$ negative to $t^{(s)}$,

$$
t=-t^{(s)}
$$

on the crack surface in order to cancel the traction due to the fundamental solution. We use the WCSE to obtain the image term. On the upper crack surface the condition (21) is written, from (17), by

$$
t_{x}^{(s)}+i t_{y}^{(s)}=\frac{2 \mu}{a(\kappa+1)} \sum_{m=1}^{M} m \delta^{(m)} U_{m-1}(X) \quad(|X| \leq 1) .
$$

Note that the minus sign of the traction is canceled by the negative unit normal $n_{y}=-1$ of the upper crack surface.

As an example, look at the uniform remote loading $\left(\sigma_{y y}^{\infty}, \sigma_{x y}^{\infty}\right)$. If we use only one term $(m=1)$ in the approximation $(9)$, then $(22)$ becomes

$$
\sigma_{x y}^{\infty}+i \sigma_{y y}^{\infty}=\frac{2 \mu \delta^{(1)}}{a(\kappa+1)} \quad(|X| \leq 1)
$$

which determines $\delta^{(1)}$. Using this result the stress intensity factors are calculated by (19) to give

$$
K( \pm 1)=K_{I}( \pm 1)+i K_{I I}( \pm 1)=\sqrt{\pi a}\left(\sigma_{y y}^{\infty}+i \sigma_{x y}^{\infty}\right)
$$

Note that the results $K_{I}( \pm 1)=\sqrt{\pi a} \sigma_{y y}^{\infty}$ and $K_{I I}( \pm 1)=\sqrt{\pi a} \sigma_{y x y}^{\infty}$ agrees with the analytical solution.

Unlike the remote uniform loading, the crack surface traction loading for the fundamental solution is not constant and $M$ multiple terms are required for the interpolation (9) of the COD. These $M$ coefficients are determined by selecting $M$ collocation points on the crack surface. Notice that the traction on the crack surface is bounded at the crack tip as seen from (17). The best result is obtained 
by including the crack tips among the collocation points. Contrast this with the numerical quadrature for the singular integral equations that must avoid the crack tip as a collocation point $[22,23]$. Once the COD coefficients are obtained, the stress intensity factors are calculated directly by the formula (19) without the need for indirect means in the post-processing.

\subsubsection{Numerical Green's function}

Consider the line force $f$ or dislocation $b$ applied at $\xi$ in an infinite domain with a crack. Once the crack opening displacement coefficients $\delta^{(m)}$ are determined, following the procedure described in Section 4.1.1, we get the Green's functions for displacement, stress and traction,

$$
\begin{aligned}
u^{(\text {total })}(z, \xi) & =u^{(s)}(z, \xi)+\sum_{m=1}^{M}\left(K^{(m)}(Z) \delta^{(m)}+\overline{L^{(m)}(Z) \delta^{(m)}}\right) \\
\sigma^{(t o t a l)}(z, \xi) & =\sigma^{(s)}(z, \xi)+\sum_{m=1}^{M}\left(P^{(m)}(Z) \delta^{(m)}+\overline{Q^{(m)}(Z) \delta^{(m)}}\right) \\
\tau^{(\text {total })}(z, \xi) & =\tau^{(s)}(z, \xi)+\sum_{m=1}^{M}\left(R^{(m)}(Z) \delta^{(m)}+\overline{S^{(m)}(Z) \delta^{(m)}}\right) \\
t^{(t o t a l)}(z, \alpha ; \xi) & =t^{(s)}(z, \alpha ; \xi)+\sum_{m=1}^{M}\left(K^{*(m)}(Z, \alpha) \delta^{(m)}+\overline{L^{*(m)}(Z, \alpha) \delta^{(m)}}\right)
\end{aligned}
$$

where the singular terms $u^{(s)}(z, \xi), \ldots, t^{(s)}(z, \xi)$ are defined by (1)-(4) and the functions $K^{(m)}(Z), \ldots, L^{*(m)}(Z, \alpha)$ by $(11)-(16)$. The stress intensity factors are still given by (19). Note that the image terms for each quantity remain the same as given in $(25)$ for the line force and dislocation.

\subsection{Single inclined crack}

Consider a crack of length $2 a_{1}$ centered at $O_{1}=z_{1}=x_{1}+i y_{1}$ with the slope $\theta_{1}$. Introduce the local coordinate system $O_{1}-x_{1} y_{1}$ with the $x_{1}$-axis along the crack and $z_{1}=x_{1}+i y_{1}$ is the local complex variable, which is related to the global $z=x+i y$ complex variable by $z_{1}=\left(z-z_{1}\right) e^{-i \theta_{1}}$. If we introduce the normalized local complex variable

$$
Z_{1}=\frac{z_{1}}{a_{1}}=\frac{z-z_{1}}{a_{1} e^{i \theta_{1}}}
$$

then the local displacement, stress and traction components are given by (11)(16) with the argument $Z_{1}$ replacing $Z$. The crack opening displacement (9), now denoted by $\delta_{1}^{(m)}$, is also defined locally. Once the crack opening displacement is 
determined locally, following the procedure described in Section 4.1.1, the transformation back to the global components will give the numerical Green's functions

$$
\begin{aligned}
u^{(\text {total })}(z, \xi) & =u^{(s)}(z, \xi)+e^{i \theta_{1}} \sum_{m=1}^{M}\left(K^{(m)}\left(Z_{1}\right) \delta_{1}^{(m)}+\overline{L^{(m)}\left(Z_{1}\right) \delta_{1}^{(m)}}\right) \\
\sigma^{(\text {total })}(z, \xi) & =\sigma^{(s)}(z, \xi)+\sum_{m=1}^{M}\left(P^{(m)}\left(Z_{1}\right) \delta_{1}^{(m)}+\overline{Q^{(m)}\left(Z_{1}\right) \delta_{1}^{(m)}}\right) \\
\tau^{(\text {total })}(z, \xi) & =\tau^{(s)}(z, \xi)+e^{-2 i \theta_{1}} \sum_{m=1}^{M}\left(R^{(m)}\left(Z_{1}\right) \delta_{1}^{(m)}+\overline{S^{(m)}\left(Z_{1}\right) \delta_{1}^{(m)}}\right) \\
t^{(\text {total })}(z, \alpha ; \xi) & =t^{(s)}(z, \alpha ; \xi)+e^{i \theta_{1}} \sum_{m=1}^{M}\left(K^{*(m)}\left(Z_{1}, \alpha-\theta_{1}\right) \delta_{1}^{(m)}+\overline{L^{*(m)}\left(Z_{1}, \alpha-\theta_{1}\right) \delta_{1}^{(m)}}\right)
\end{aligned}
$$

where the singular terms are defined by (1)-(4). Note that the traction is calculated along a line segment at $z$ with the unit normal $n_{x}+i n_{y}=e^{i \alpha}$ in the global system. The angle $\alpha$ becomes $\alpha-\theta_{1}$ in the local coordinate system.

\subsection{Multiple center cracks}

The scheme developed above for the Green's functions for the single crack can readily be extended to multiple cracks. For each of the multiple cracks we introduce the local coordinate system, $O_{j}-x_{j} y_{j}(j=1, \ldots, N)$ in which the $x_{j}$-axis is aligned with the crack. In addition, the global coordinate system $O-x y$ is used to specify the crack geometry by its center $z_{j}$, inclination $\theta_{j}$, and length $a_{j}$. The traction contribution from each crack is calculated by (15) or (17) in the local system. The unknown crack opening coefficients $\delta_{j}^{(m)}$ defined locally by $(9)$ for each crack. The numerical green's function for the traction has the form

$$
\begin{aligned}
& t^{(\text {total })}(z, \alpha ; \xi)=t^{(s)}(z, \alpha ; \xi) \\
+ & \sum_{j=1}^{N} e^{i \theta_{j}} \sum_{m=1}^{M_{j}}\left(K^{*(m)}\left(Z_{j}, \alpha-\theta_{j}\right) \delta_{j}^{(m)}+\overline{L^{*(m)}\left(Z_{j}, \alpha-\theta_{j}\right) \delta_{j}^{(m)}}\right),
\end{aligned}
$$

where $M_{j}$ is the number of terms in the approximation (9) for each crack. Since the unit normal to the crack $k$ has an angle $\alpha_{k}=\theta_{k}-\pi / 2$, the zero traction condition on this crack is given by

$$
\sum_{j=1}^{N} e^{i \theta_{j}} \sum_{m=1}^{M_{j}}\left(K^{*(m)}\left(Z_{j}, \alpha_{k}-\theta_{j}\right) \delta_{j}^{(m)}+\overline{L^{*(m)}\left(Z_{j}, \alpha_{k}-\theta_{j}\right) \delta_{j}^{(m)}}\right)=-t^{(s)}\left(z, \alpha_{k} ; \xi\right),
$$

which is evaluated at $M_{k}$ sample points to determine the unknown crack opening displacement coefficients. Notice that, in (29), the traction contribution from the 
crack $k$ itself should be calculated by formula (17). Once $\delta_{j}^{(m)}$ are determined the Green's functions are given by

$$
\begin{aligned}
u^{(t o t a l)}(z, \xi) & =u^{(s)}(z, \xi)+\sum_{j=1}^{N} e^{i \theta_{j}} \sum_{m=1}^{M_{j}}\left(K^{(m)}\left(Z_{j}\right) \delta_{j}^{(m)}+\overline{L^{(m)}\left(Z_{j}\right) \delta_{j}^{(m)}}\right), \\
\sigma^{(t o t a l)}(z, \xi) & =\sigma^{(s)}(z, \xi)+\sum_{j=1}^{N} e^{i \theta_{j}} \sum_{m=1}^{M_{j}}\left(P^{(m)}\left(Z_{j}\right) \delta_{j}^{(m)}+\overline{Q^{(m)}\left(Z_{j}\right) \delta_{j}^{(m)}}\right),(30) \\
\tau^{(t o t a l)}(z, \xi) & =\tau^{(s)}(z, \xi)+e^{-2 i \theta_{1}} \sum_{j=1}^{N} e^{i \theta_{j}} \sum_{m=1}^{M_{j}}\left(R^{(m)}\left(Z_{j}\right) \delta_{j}^{(m)}+\overline{S^{(m)}\left(Z_{j}\right) \delta_{j}^{(m)}}\right),
\end{aligned}
$$

along with (28). Note that (28) and (30), with the proper singular terms, give the universal form for the numerical Green's functions for any fundamental solutions and their combinations including their continuous distributions in the sense that the form of the image terms remains the same.

\section{Error analysis of single crack}

\subsection{Crack surface traction by the fundamental solutions}

Consider a crack $(-a, a)$ on the $x$-axis. We normalize the coordinates by half crack length $a$, such that the crack is located in the interval $(-1,1)$ of the $x$-axis. We have calculated the traction along the prospective crack surface, in an un-cracked domain, generated by the fundamental solutions. The fundamental solutions with the unit magnitude in $x$ and $y$ directions, respectively, are placed along the $y$-axis (center line of the crack) at $y=0.1,1.0$ and 5.0. Figure 1 shows the traction $t_{x}$ and $t_{y}$ distributions in the interval $(-1,1)$ due to the unit horizontal line force $f_{x}=1$. The corresponding traction components for the vertical unit line force $f_{y}=1$ are given in Figure 2. Notice the peak and the double peaks of the distributions for the line force at $y=0.1$. Similar features can be observed for the traction distribution due to the unit line dislocation with $b_{x}=1$ (as shown in Figure 3 ) and $b_{y}=1$ (not shown) for the dislocation.

It is well known that the $p$-type interpolation, like (9), does not approximate these peaks well. Thus, we expect error in the results when the singularities approach the crack. It is the objective of this section to assess the error and identify the domain of applicability of the numerical Green's functions where the error is small.

\subsection{Stress intensity factors by analytical Green's function}

The dimensional quantities appearing in the problem are the half crack length $a(m)$, shear modulus $\mu\left(N / m^{2}\right)$, magnitudes of the line force $f(N / m)$ and dislocation $b(m)$. We select the typical magnitudes of the non-dimensional $b$ and $f$ to be 

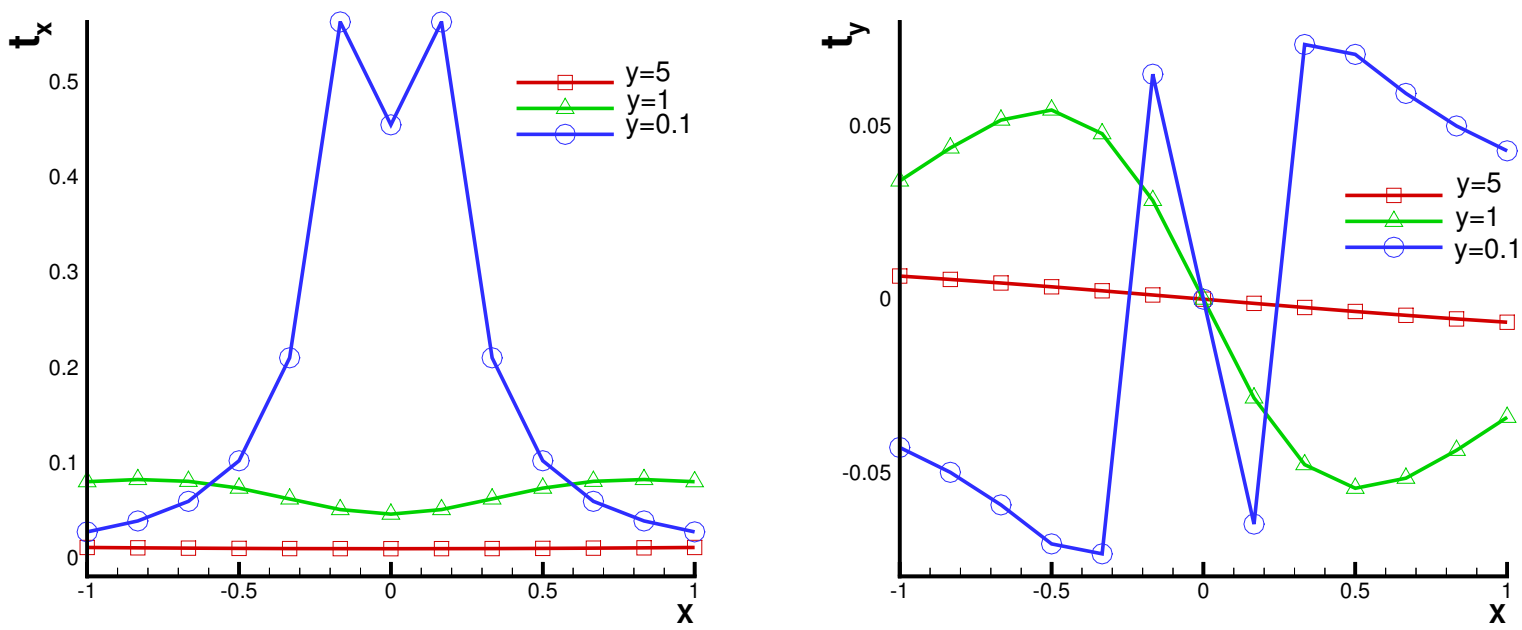

Figure 1: Crack line traction by the horizontal line force.

$\bar{b}=b / a=10^{-2}$ and $\bar{f}=\bar{\mu} \bar{b}=10^{-2} \bar{\mu}$, where the non-dimensional shear modulus is selected to be $\bar{\mu}=1$ to give $\bar{f}=10^{-2}$. In this section, the bar indicates the nondimensional quantity. We have calculated the stress intensity factors for the unit components $\left(\bar{f}_{x}, \bar{f}_{y}\right)$ and $\left(\bar{b}_{x}, \bar{b}_{y}\right)$ of the line force and dislocation, respectively, in the unit of $10^{-2}$. The analytical Green's functions, which automatically satisfy the crack surface traction free condition, described in Appendix B are used. Figures 4 - 5 show the variation of the stress intensity factors $K_{I}$ and $K_{I I}$ as the function of the location of the fundamental solutions. We have placed the unit line force components $\bar{f}_{x}$ and $\bar{f}_{y}$ and line dislocation components $\bar{b}_{x}$ and $\bar{b}_{y}$, respectively, at the location $(x, y)$ to calculate the resulting stress intensity factors (SIFs) at the right crack tip $x=+1$. By changing the location we have calculated the SIFs as the function of the location and plotted in these Figures. Notice the regions of near zero SIF, away from the crack, in Figure 4 for $K_{I}$ by $\bar{f}_{x}$ and $K_{I I}$ by $\bar{f}_{y}$. The line dislocation components $\bar{b}_{x}$ and $\bar{b}_{y}$ have regions of low SIFs for both $K_{I}$ and $K_{I I}$ as shown in Figure 5. This is the region where we expect the numerical Green's functions to have difficulties in addition to the near crack region mentioned earlier.

\subsection{Comparison of analytical/numerical Green's functions}

To support the speculation on the domains of high error expected of the numerical Green's functions and identify the domains where such error is low, we have performed the error analysis. We have calculated the SIF by the numerical and analytical Green's functions and obtained the relative error in the SIFs as the 

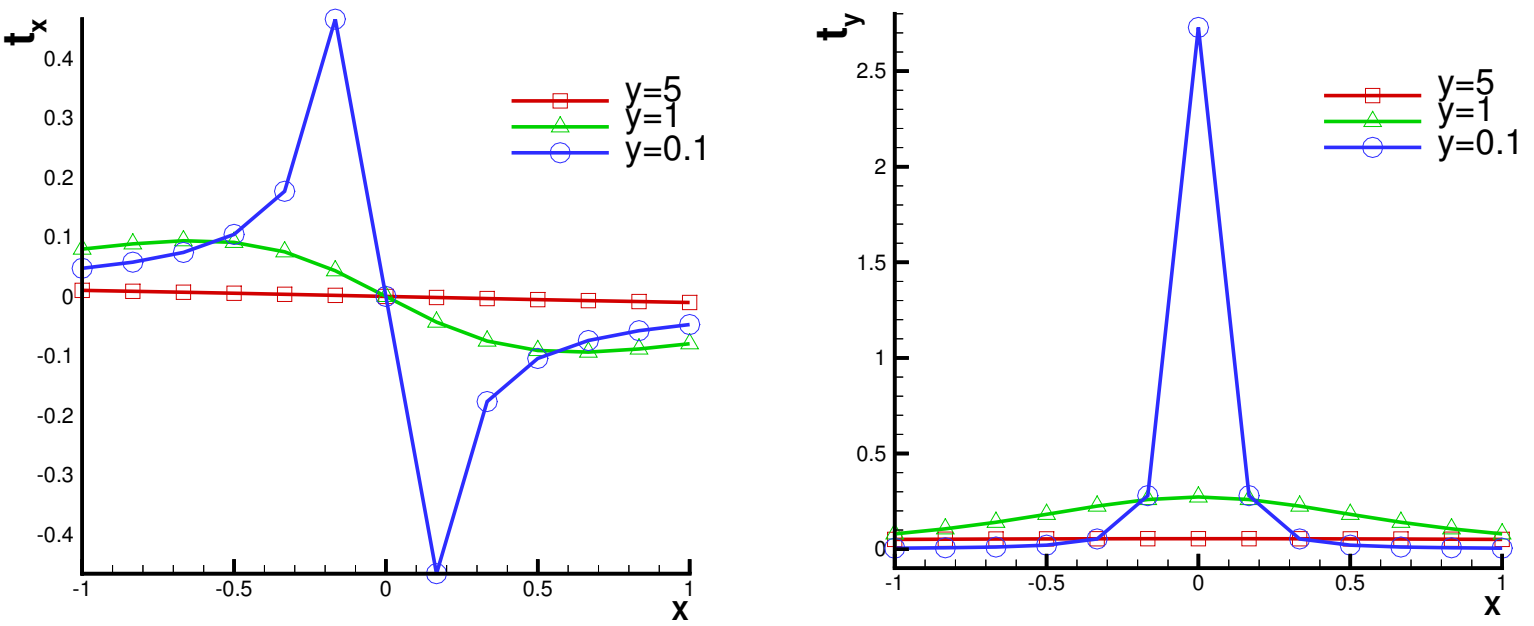

Figure 2: Crack line traction by the vertical line force.

function of the location of the fundamental solutions. The procedure is the same as the SIF calculation by the analytic Green's function discussed above. The numbers of the terms used in the interpolation (9) are $M=3,7$ and 14. Each plot in the Figures 6 - 9 has three contours marking the boundaries of $0.1 \%, 1 \%$ and $10 \%$ relative errors, respectively. For example the region outside the $0.1 \%$ error contour has the relative error less than $0.1 \%$. The region inside the $10 \%$ error contour has the error more than $10 \%$.

In all of these Figures, we see the error near the crack is unacceptably high, which is indicated the domain inside the $10 \%$ error contour. For $M=3$, the contour of $0.1 \%$ error is typically located in the circle with the radius $10 a$ (not shown), where $a$ is the half crack length. The rectangular boxes marked at distance $2 a$ and $a$ apart from the crack approximately represents the $1 \%$ and $10 \%$ error contours, respectively. For $M=7$, the accuracy improves. The $0.1 \%$ and $1 \%$ error contours are closely represented by the rectangular boxes marked at distances $2 a$ and $a$, respectively. For $M=14$, however, while the $0.1 \%$ and $1 \%$ error contours remain the same as for $M=7$, the contour of $10 \%$ error expands away from the crack. The use of more terms in the approximation does not improve the accuracy of the results near the crack, rather the accuracy deteriorates. This is the typical behavior of the $p$-type interpolation when it encounters peaks in the approximation region.

In addition to the severely high error region near the crack, we also have observed regions of mildly high error away from the crack. As speculated earlier, we have confirmed that these are the regions of near zero SIFs. The numerical 

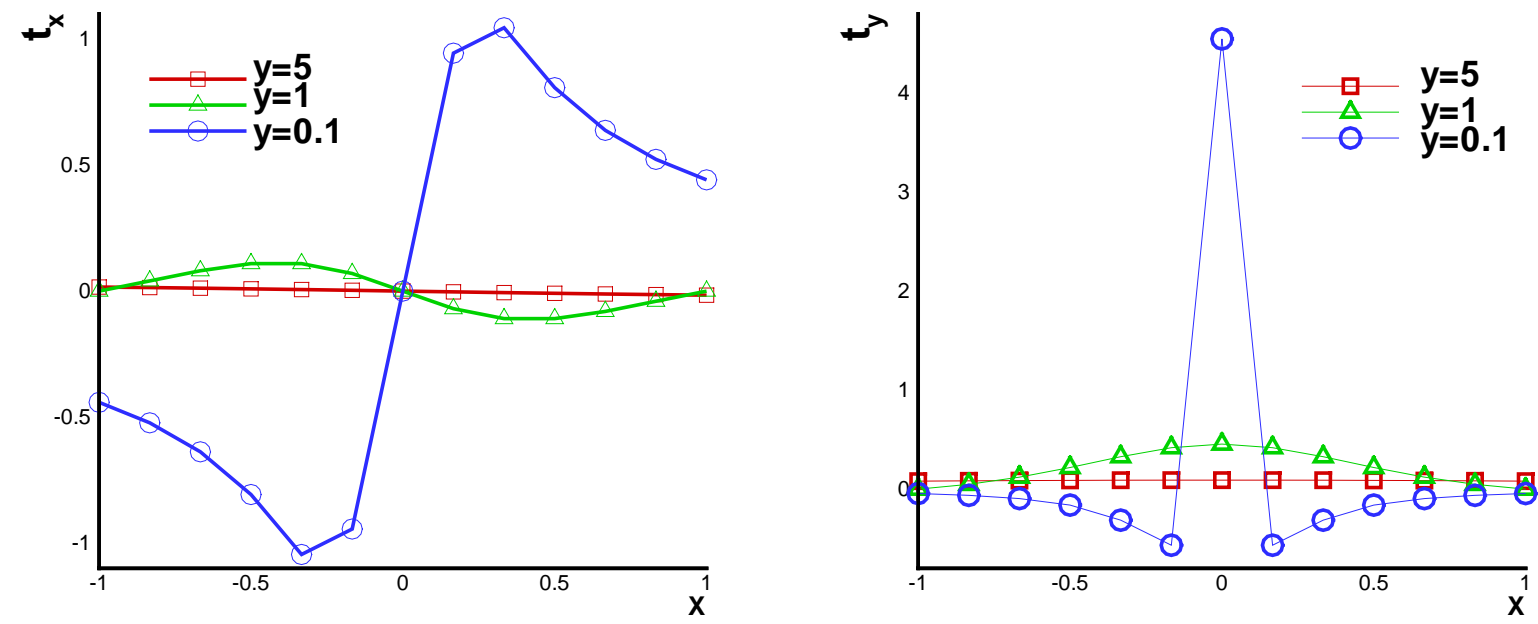

Figure 3: Crack line traction by line dislocation $b_{x}=1$.

Green's functions have difficulty in approximating near zero SIFs.

Identification of the zone of high error by the numerical Green's functions serves as the guideline in their applications. The typical application is for the boundary element method (BEM), where the fundamental solutions are distributed along the boundary and integrated. In the process of integration, the order of the singularity of these fundamental solutions drops to an extent that we can place the integrated fundamental solutions (i.e., layer potentials) at the distance closer to the crack than the discrete singularities without severe errors. For the layer potentials, the recommended approach is to integrate the fundamental solutions analytically in an infinite domain without the crack and use the numerical Green's function technique for the layer potentials. This way we are dealing with less singular entities than the original fundamental solution. Nevertheless, it is important to improve the near crack behavior of the proposed numerical Green's functions. Such numerical Green's functions are under investigation. 


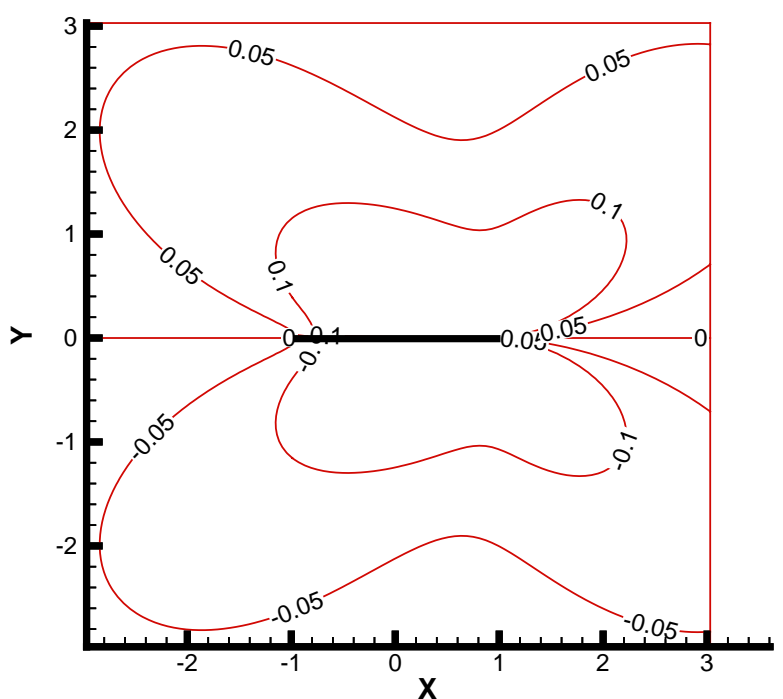

(a)

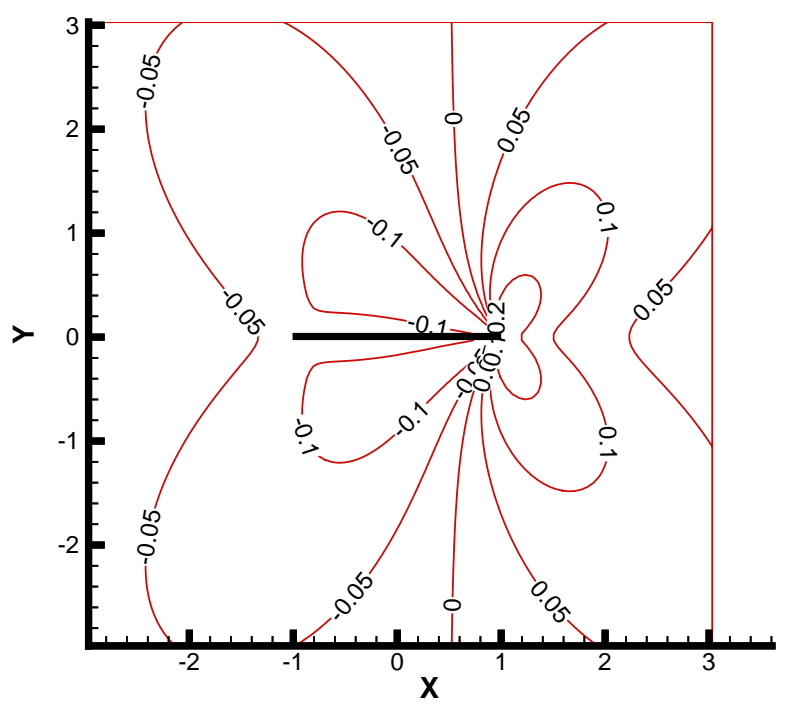

(c)

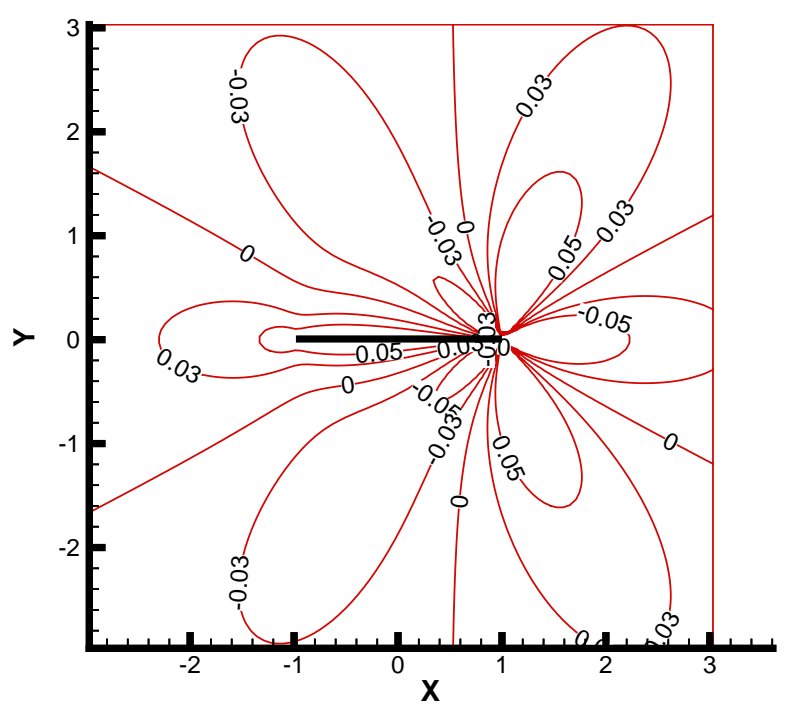

(b)

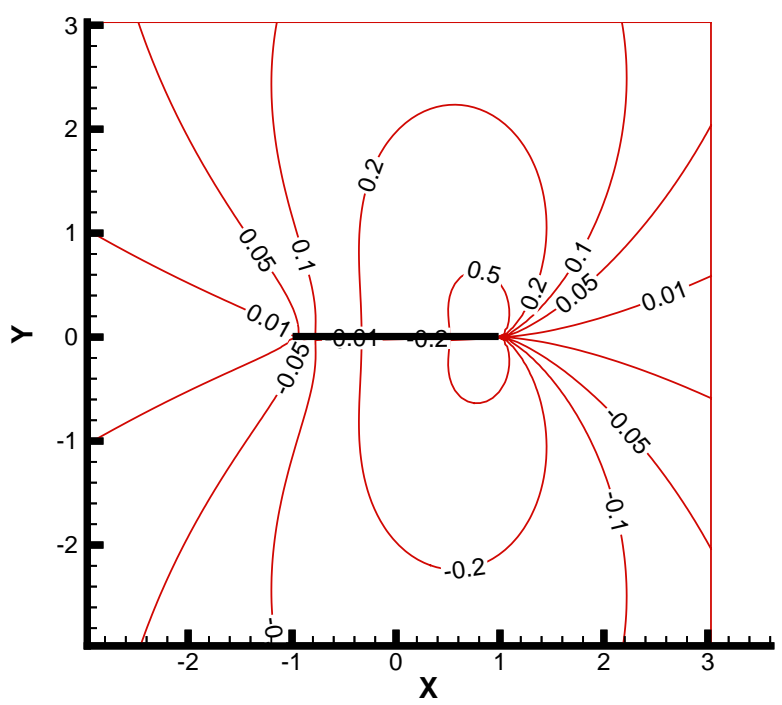

(d)

Figure 4: Variation of the SIFs as the function of the location $(x, y)$ of the unit line forces. (a) $K_{I I}$ by $f_{x}=10^{-2}$, (b) $K_{I}$ by $f_{x}=10^{-2}$, (c) $K_{I I}$ by $f_{y}=10^{-2}$, (b) $K_{I}$ by $f_{y}=10^{-2}$. Analytical solution. 


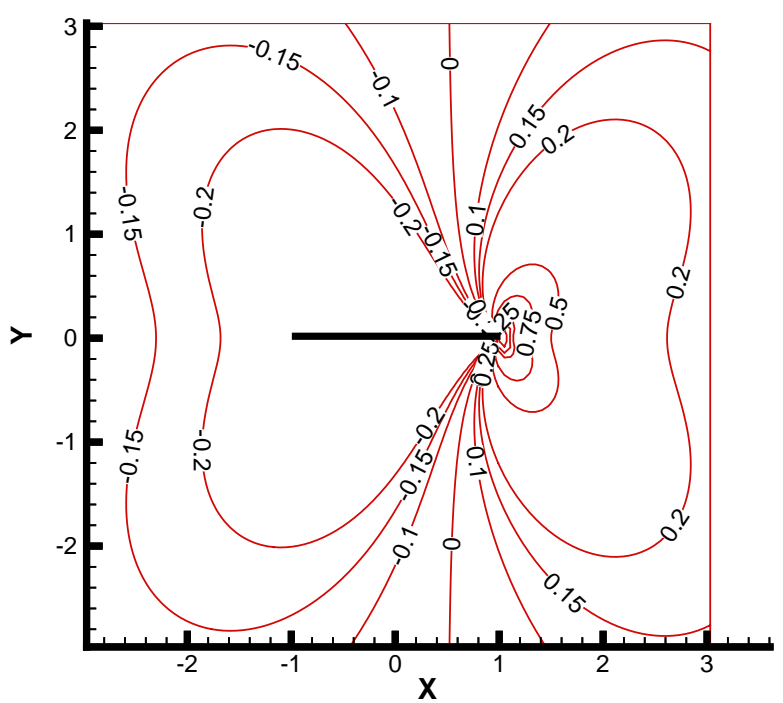

(a)

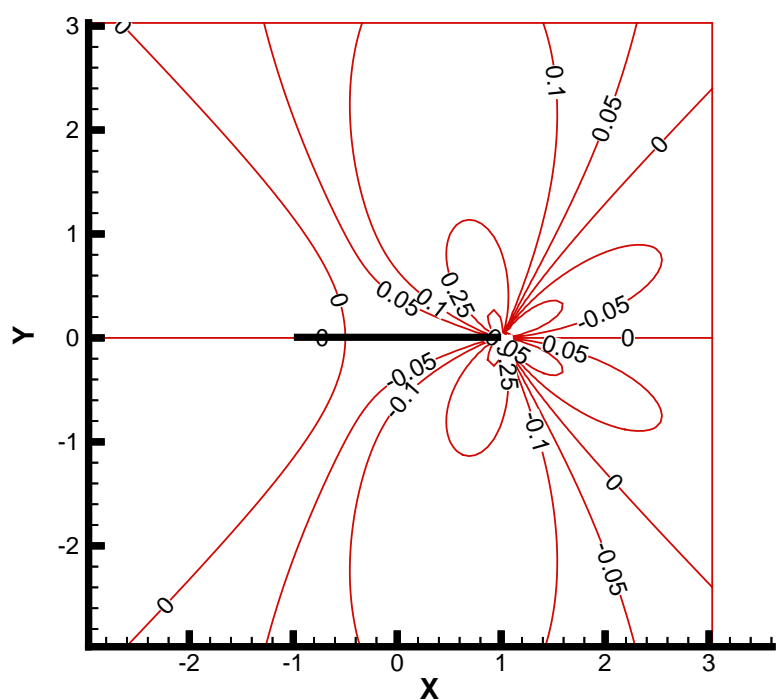

(c)

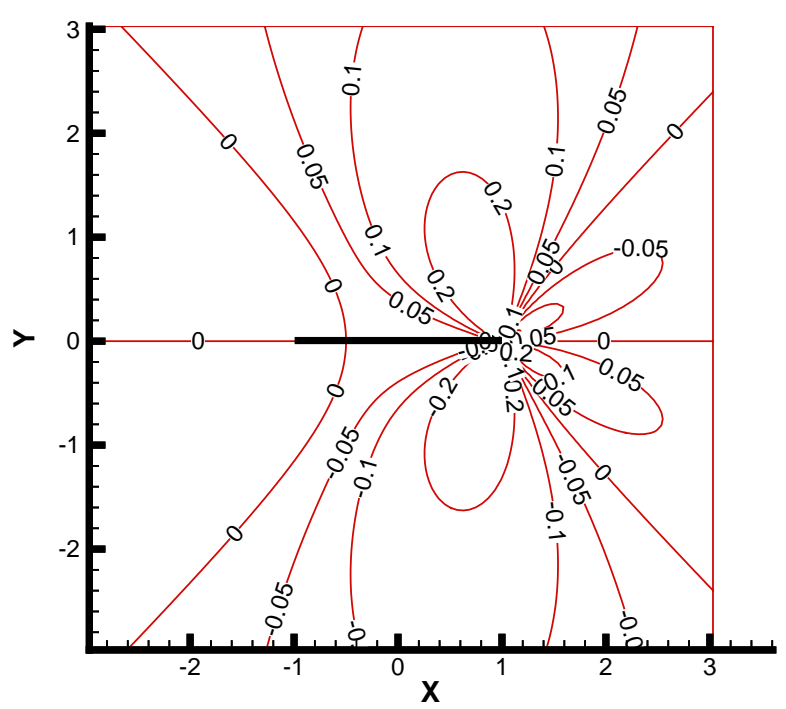

(b)

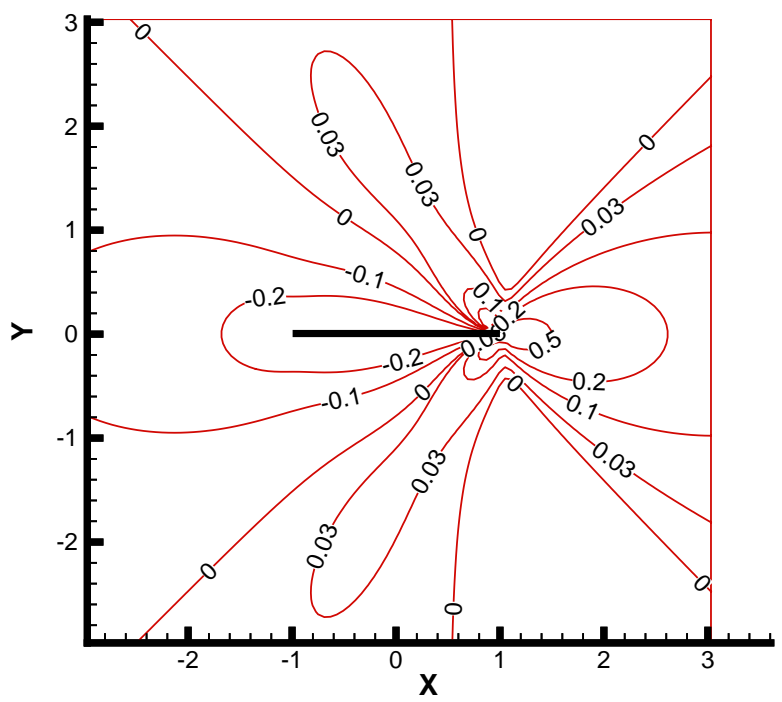

(d)

Figure 5: Variation of the SIFs as the function of the location $(x, y)$ of the unit line dislocations. (a) $K_{I I}$ by $b_{x}=10^{-2}$, (b) $K_{I}$ by $b_{x}=10^{-2}$, (c) $K_{I I}$ by $b_{y}=10^{-2}$, (b) $K_{I}$ by $b_{y}=10^{-2}$. Analytical solution. 


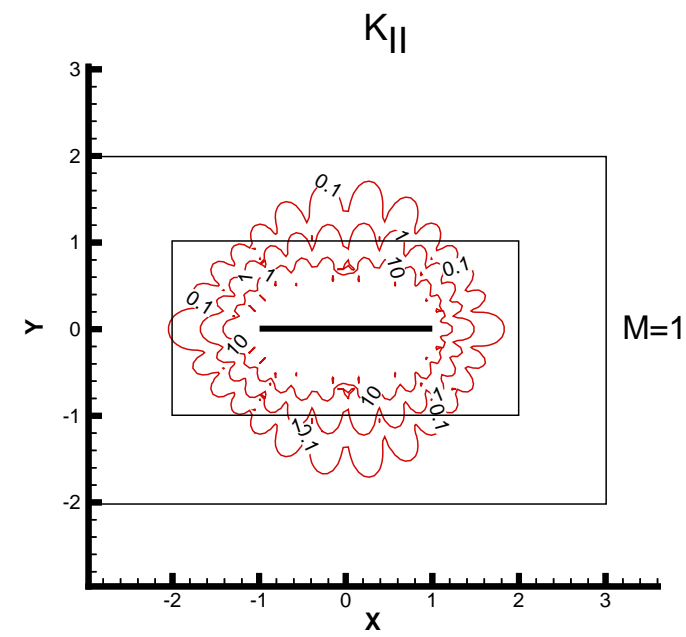

$\mathrm{K}_{\mathrm{l}}$
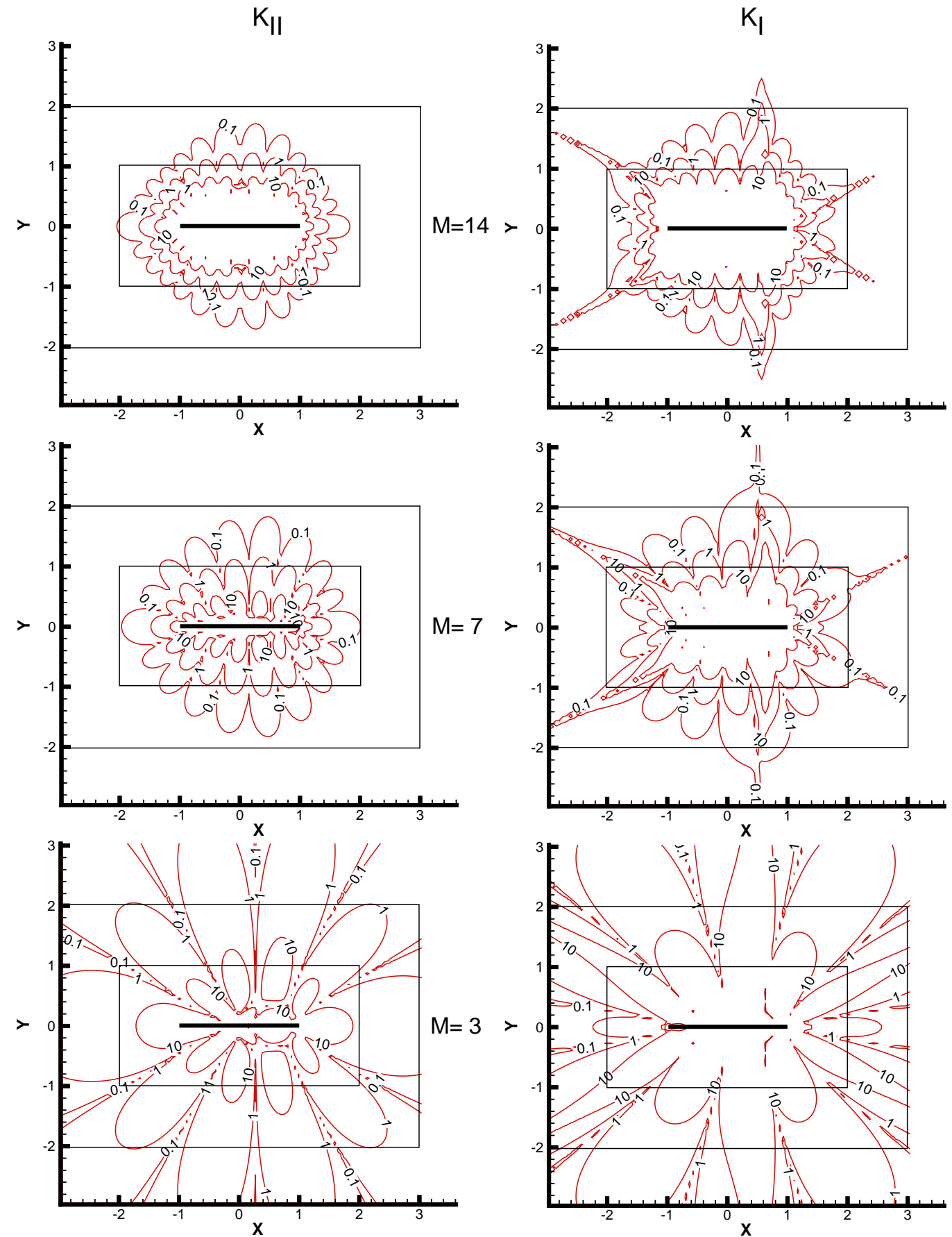

Figure 6: Variation of the relative error in $K_{I}(+1)$ and $K_{I I}(+1)$ as the function of the location $(x, y)$ of the unit line force $f_{x}$. Number of polynomials used are $\mathrm{M}=3,7,14$. 

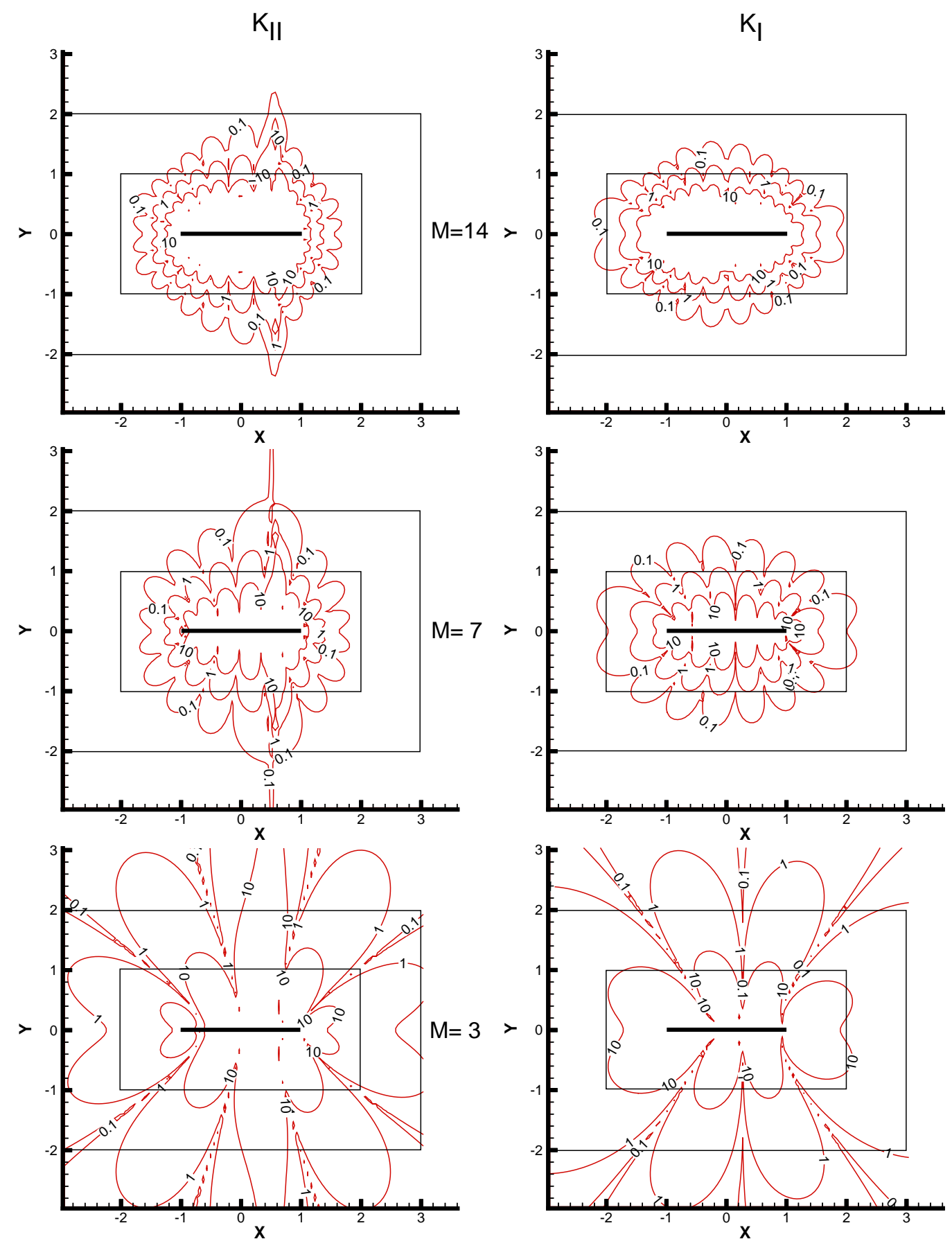

Figure 7: Variation of the relative error in $K_{I}(+1)$ and $K_{I I}(+1)$ as the function of the location $(x, y)$ of the unit line force $f_{y}$. Number of polynomials used are $\mathrm{M}=3,7,14$. 


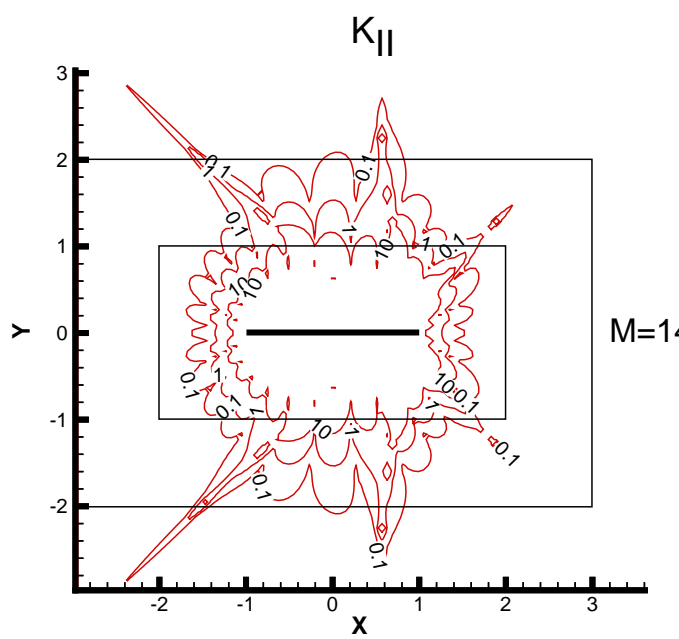

$\mathrm{K}_{\mathrm{I}}$
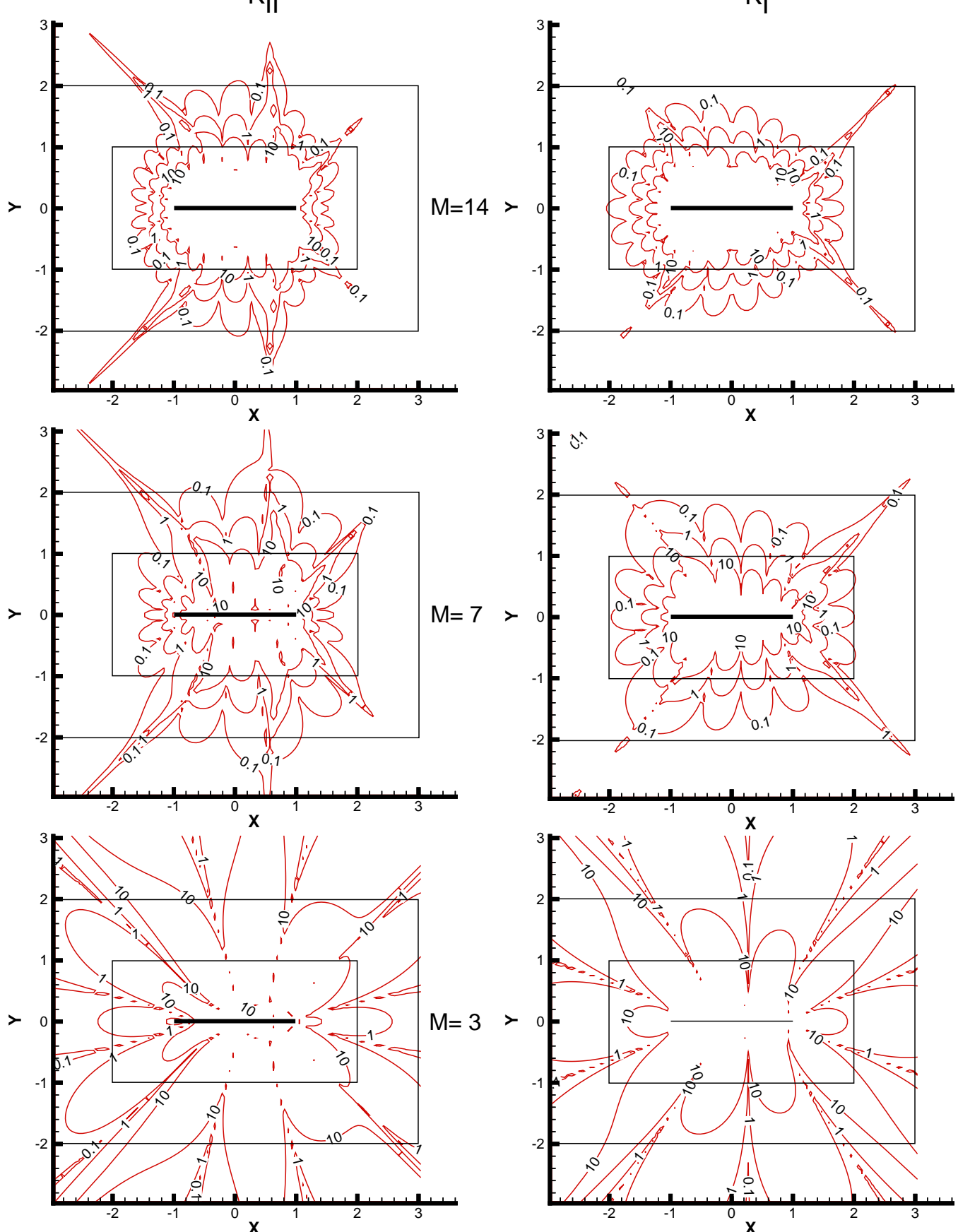

Figure 8: Variation of the relative error in $K_{I}(+1)$ and $K_{I I}(+1)$ as the function of the location $(x, y)$ of the unit line force $b_{x}$. Number of polynomials used are $\mathrm{M}=3,7,14$. 

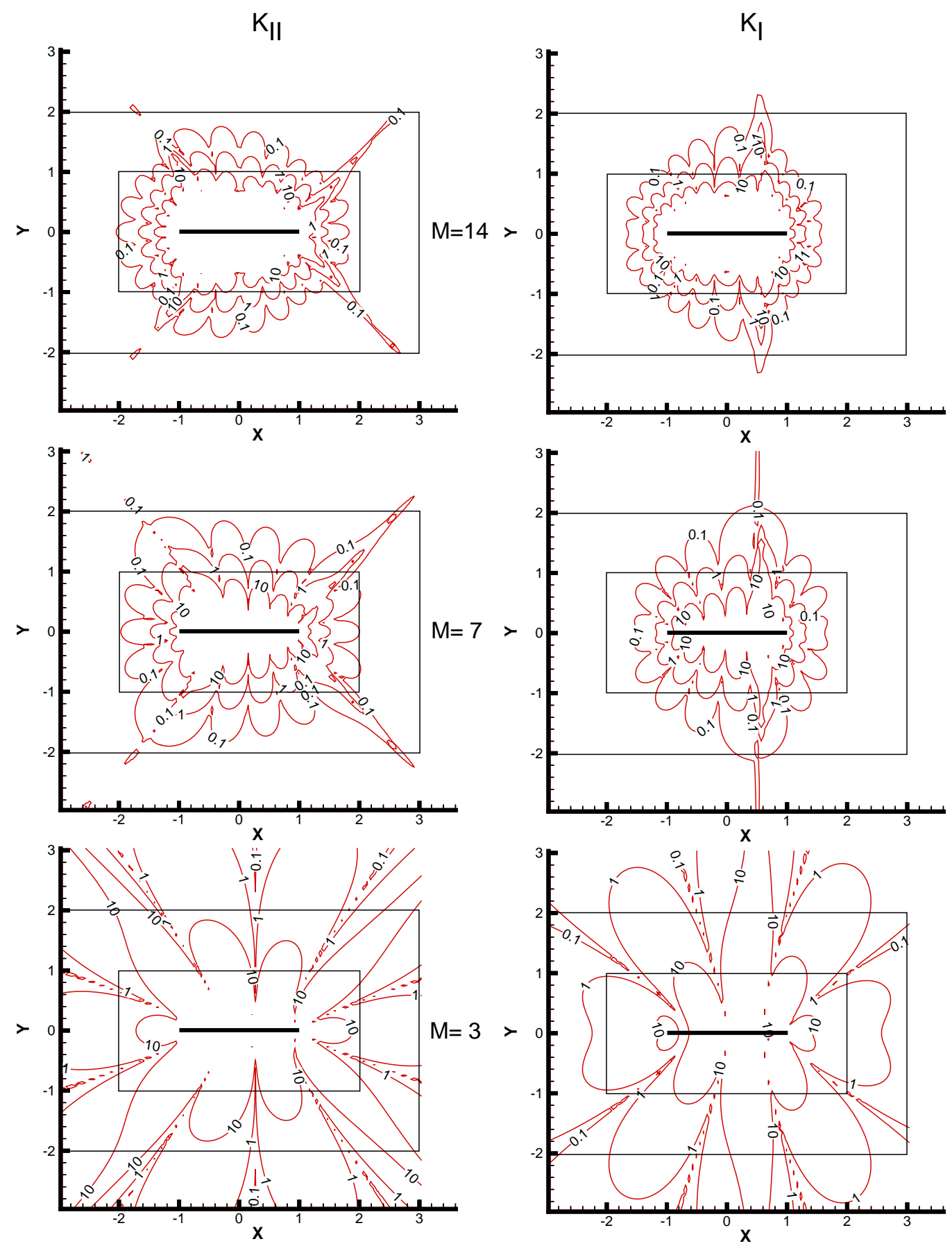

Figure 9: Variation of the relative error in $K_{I}(+1)$ and $K_{I I}(+1)$ as the function of the location $(x, y)$ of the unit line force $b_{y}$. Number of polynomials used are $\mathrm{M}=3,7,14$. 


\section{$6 \quad$ Numerical Green's functions for Multiple cracks}

Following the guideline set by the error analysis for a single crack, numerical Green's functions for three multiple crack configurations are obtained for the line force and dislocation. The characteristic length in each problem is the half crack length $a$ with which the problem is non-dimensioned. The non-dimensional shear modulus $\bar{\mu}=2$ and Poisson's ratio $\nu=0.3$ are used. The collinear straight center crack configuration places the cracks on the intervals $(-3,-1)$ and $(+1,+3)$ along the $x$-axis. The parallel straight center crack configuration centers the cracks on the $y$-axis on the interval $(-1,+1)$ along the lines $y=-1$ and $y=+1$. The inclined straight center crack configuration places the crack tips at the coordinates $(-1.7071,-0.7071)$ and $(-0.2929,0.7071)$ for one crack, giving a crack inclination of $\alpha=30^{\circ}$ and maintaining a half crack length of $a=1$ as is the case in the two previous configurations. The second crack in the inclined crack configuration is mirrored from the first along the $y$-axis. Each crack configuration is loaded at $y=5.0$ on the $\mathrm{y}$-axis with four unit non-dimensional loading cases, $\overline{b_{x}}=1, \overline{b_{y}}=1$, $\bar{f}_{x}=1, \bar{f}_{y}=1$. This location maintains the position outside the $2 a$ rectangular region surrounding each of the multiple cracks associated with less than $0.1 \%$ error region and avoiding the mildly high error regions extending away from the crack. The order of the polynomials is set at $M=7$ for the interpolation. The resulting stress and displacement fields are plotted on a square 2-D contour plot with an interval of $(-4,+4)$ for $x$ and $y$ (placing the loading outside the plotted region). The contours are individually labeled and a color coded legend is provided. A 3-D shaded plot with the $z$-axis set as the component value, accompanies the 2-D plot. The complete set of figures can be found in Quick's M.S. thesis [24] and we list some of the most interesting cases in this paper. Results for the line force with two collinear cracks are in excellent agreement with the analytical solution obtained by Erdogan [25]. There are no analytical solutions available for other crack and loading configurations.

\section{Conclusion}

We have proposed the numerical Green's functions for multiple cracks based on the whole crack singular element. The Green's functions have the $\sqrt{r}$ to eliminate the need for the post processing for the stress intensity factor calculation. The crack opening and $1 / \sqrt{r}$ crack tip stress singularity are embedded mathematically. However, the $p$-type approximation used has the weakness in capturing the near crack behavior of the fundamental solutions that give rise to steep variation of the crack surface traction to be dealt with the image term of the Green's functions. For the single crack, we have identified the domains of high error as well as the domains where the numerical green's functions can be used reliably. Following the guideline set by the error analysis to avoid the near crack region of high error, the actual numerical Green's functions can be obtained for arbitrary straight multiple crack configurations. The complete listing of the numerical Green's functions 
for the three configurations of the multiple crack treated in this paper is given by Quick [24]. The effort to improve the near crack behavior of the numerical Green's functions is in progress.

\section{Acknowledgment}

P. Quick was supported by the NSF IGERT Grant under subcontract from the University of Tennessee, Knoxville.

\section{References}

[1] J. Telles, G. Castor and S. Guimarães, A numerical Green's function apparoach for boundary elements applied to fracture mechanics, Int. J. Num. Meth. Engng., vol. 38, pp. 3259-3274, 1995.

[2] N. Silveira, S. Guimarães and J. Telles, Accurate hypersingular integral computations in the development of numerical Green's function for fracture mechan$i c s$, chap. 6, Singulr Integrals in Boundary Element Methods, Computational Mechanics Publication, Southampton, 1998.

[3] G. Castor and J. Telles, The 3-D BEM implementation of a numerical Green's function for fracture mechanics application, Int. J. Num. Meth. Engng., vol. 48, pp. 1199-1214, 2000.

[4] M. D. Snyder and T. A. Cruse, Boundary integral equation analysis of cracked anisotropic plates, Int. J. Frac., vol. 11, pp. 315-328, 1975.

[5] T. A. Cruse, Two-dimensional bie fracture mechanics analysis, Appl. Math. Modelling, vol. 2, pp. 287-292, 1978.

[6] D. L. Clements and M. D. Haselgrove, A boundary integral equation method for a class of crack problems in anisotropic elasticity, Int. J. Comp. Math., vol. 12, pp. 267-278, 1983.

[7] J. R. Berger and V. K. Tewary, Boundary integral equation formulation for interface cracks in anisotropic materials, Int. J. Numerical Methods Engng., vol. 20, pp. 261-266, 1997.

[8] R. Yuuki and S. Cho, Efficient boundary element analysis of stress intensity factors for interface cracks in dissimilar materials, Engng. Fract. Mech., vol. 30, pp. 179-188, 1989.

[9] M. Morjaria and S. Mukherjee, Numerical analysis of planar time dependent inelastic deformation of plates with cracks by the boundary element method, Int. J. Solids Structures, vol. 17, pp. 127-143, 1981. 
[10] W. T. Ang and C. D. L., A boundary element method for determining the effect of holes on the stress distribution around a crack, Int. J. Numer. Meth. Engng., vol. 23, pp. 1727-1737, 1986.

[11] M. Kamel and B. M. Liaw, Boundary element formulation with special kernels for an anisotropic plate containing an elliptical hole or a crack, Engng. Fracture Mech., vol. 39(4), pp. 695-711, 1991.

[12] C. Hwu and Y. W. J., Green's functions of two-dimensional anisotropic plates containing an elliptic hole, Int. J. Solids Structures, vol. 27(13), pp. 17051719, 1991.

[13] M. Denda and I. Kosaka, Dislocation and point-force-based approach to the special green's function bem for elliptic hole and crack problems in two dimensions, Int. J. Numerical Methods in Engineering, vol. 40, pp. 2857-2889, 1997.

[14] J. C. F. Telles and C. A. Brebbia, Boundary element solution for half-plane problems, Int. J. Solids Structures, vol. 17, pp. 1149-1158, 1981.

[15] J. Meek and C. Dai, Boundary element modeling: Near surface excavations, Comp Meth. Appl. Mech. Eng., vol. 102, pp. 15-27, 1993.

[16] P. Dumir and A. Mehta, Boundary element solution for elastic orthotropic half-plane problems, Computers and Structures, vol. 26, pp. 431-438, 1987.

[17] E. Pan, C. Chen and B. Amadei, A BEM formulation for anisotropic halfplane problems, Engng. Anal. with Boundary Elements, vol. 20, pp. 185-195, 1997.

[18] J. R. Berger, Boundary element analysis of anisotropic bimaterials with special green's functions, Engng. Anal. with Boundary Elements, vol. 14(2), pp. 123-131, 1994.

[19] M. Denda, The green's function BEM for bimaterial solids applied to edge stress concentration problems, Electronic J. Boundary Elements, vol. 1(2), pp. 112-131, 2003.

[20] N. Muskhelishvili, Some Basic Problems of the Mathematical Theory of Elasticity, Noordhoff, Groningen, 1958.

[21] M. Denda and Y. F. Dong, Complex variable approach to the BEM for multiple crack problems, Compt. Methods Appl. Mech. Engrg., vol. Vol. 141, pp. pp. 247-264, 1997.

[22] F. Erdogan, G. D. Gupta and T. S. Cook, Numerical solution of singular integral equations, in Mechanics of Fracture 1: Methods of Analysis and Solutions of Crack Problems, (ed.) G. C. Sih, pp. 368-425, Noordhoff, 1973. 
[23] P. S. Theocaris and N. I. Ioakimidis, Numerical integration method for the solution of singular integral equations, Quart. Appl. Math, vol. 35, pp. 173183, 1977.

[24] P. Quick, Numerical Green's functions for line force and dislocation around multiple cracks, Master's thesis, Rutgers University, 2004.

[25] F. Erdogan, On the stress distribution in plates with collinear cuts under arbitrary loads, in Fourth U.S. National Congress of Applied Mechanics, p. 547, 1962.

[26] M. Denda, Complex variable Green's function representation of plane inelastic deformation in isotropic solids, Acta Mechanica, vol. 72, pp. 205-221, 1988.

[27] M. Denda, Formulation of the plastic source method for plane inelastic problems, part 1: Green's functions for plane inelastic deformation, Acta Mechanica, vol. 75, pp. 93-109, 1988.

[28] M. A. Jaswon and G. T. Symm, Integral Equation Methods in Potential Theory and Elastostatics, Academic Press, London, 1977.

[29] G. M. L. Gladwell and A. H. England, Orthogonal polynomial solutions to some mixed boundary-value problems in elasticity theory, Q. Jl. Mech. Appl. Math., vol. XXX, Pt. 2, pp. 175-185, 1977. 


\section{A Appendix}

\section{A.1 Muskhelishvili's complex variable formalism}

In Muskhelishvili's complex variable formalism [20] for plane isotropic elasticity, the solutions are given by two analytic functions (or complex potential functions), $\phi(z)$ and $\psi(z)$, of a complex variable $z=x+i y$, where $i=\sqrt{-1}$. The displacement $\left(u_{x}, u_{y}\right)$ and the stress $\left(\sigma_{x x}, \sigma_{y y}, \sigma_{x y}\right)$ components are given by

$$
2 \mu u \equiv 2 \mu\left(u_{x}+i u_{y}\right)=\kappa \phi(z)-z \overline{\phi^{\prime}(z)}-\overline{\psi(z)},
$$

and

$$
\begin{aligned}
\sigma \equiv \frac{\sigma_{x x}+\sigma_{y y}}{2} & =2 \operatorname{Re}\left\{\phi^{\prime}(z)\right\}, \\
\tau \equiv \frac{\sigma_{y y}-\sigma_{x x}}{2}+i \sigma_{x y} & =\bar{z} \phi^{\prime \prime}(z)+\psi^{\prime}(z),
\end{aligned}
$$

where $\mu$ is the shear modulus and Muskhelishvili's constant $\kappa$ is given by $\kappa=3-4 \nu$ in plane strain and $\kappa=(3-\nu) /(1+\nu)$ in plane stress in terms of Poisson's ratio $\nu$. The symbol $R e$ indicates the real part and a bar indicates the complex conjugate. A prime attached to the analytic functions of $z$ indicates differentiation by $z$ and a bar the complex conjugate. The traction on a line segment with the unit normal $n_{x}+i n_{y}=e^{i \alpha}$ is given by

$$
t=t_{x}+i t_{y}=2 e^{i \alpha} \operatorname{Re}\left\{\phi^{\prime}(z)\right\}-e^{-i \alpha}\left\{z \overline{\phi^{\prime \prime}(z)}+\overline{\psi^{\prime}(z)}\right\} .
$$

\section{A.2 Fundamental solutions}

\section{A.2.1 Line force and dislocation}

Consider a line force $f=f_{x}+i f_{y}$ (per unit thickness) and a line dislocation $b=b_{x}+i b_{y}$ located, independently, at $\xi$ in an infinite body. Their complex potential function solutions are given by $[26,27]$

$$
\begin{aligned}
\phi^{(s)}(z ; \xi) & =-\gamma \log (z-\xi), \\
\psi^{(s)}(z ; \xi) & =-k \bar{\gamma} \log (z-\xi)+\gamma \frac{\bar{\xi}}{z-\xi}
\end{aligned}
$$

where

$$
\left\{\begin{array}{lll}
k=-\kappa, & \gamma=f / 2 \pi(\kappa+1) & \text { line force } \\
k=1, & \gamma=i \mu b / \pi(\kappa+1) & \text { line dislocation. }
\end{array}\right.
$$

\section{A.2.2 Dipoles}

A pair of point forces $-f$ and $f$ (or a pair of edge dislocations $-b$ and $b$ ) located at an infinitesimal distant $d \xi$ apart defines a force dipole (or a dislocation dipole). 
The force dipole represents a couple and the dislocation dipole represents a displacement discontinuity over the infinitesimal line segment. The dipole solutions are given by $[26,27]$

$$
\begin{aligned}
d \phi^{(s)}(z ; \xi) & =-\gamma d\{\log (z-\xi)\}, \\
d \psi^{(s)}(z ; \xi) & =-k \bar{\gamma} d\{\log (z-\xi)\}+\gamma d\left\{\frac{\bar{\xi}}{z-\xi}\right\},
\end{aligned}
$$

where $d(\cdots)$ is the total differentiation operator

$$
d(\cdots)=\frac{\partial}{\partial \xi}(\cdots) d \xi+\frac{\partial}{\partial \bar{\xi}}(\cdots) d \bar{\xi}
$$

\section{A.2.3 Layer potentials}

Consider the continuous distribution of the line forces (or the line dislocations) over an $\operatorname{arc} L$. The complex potential functions are given by the line integral of (A.4),

$$
\begin{aligned}
\phi^{(s)}(z) & =-\int_{L} \Gamma(s) \log (z-\xi) d s, \\
\psi^{(s)}(z) & =-k \int_{L} \overline{\Gamma(s)} \log (z-\xi) d s+\int_{L} \Gamma(s) \frac{\bar{\xi}}{z-\xi} d s,
\end{aligned}
$$

where $\Gamma(s)$ is the density function representing the traction (or the dislocation gradient) per unit length of the arc with $s$ being the arc length. The complex potential functions for the continuous distribution of force dipoles (or the dislocation dipoles) over $L$ are given by the line integral of (A.6),

$$
\begin{aligned}
\boldsymbol{d} \phi^{(s)}(z) & =-\int_{L} \gamma(s) d\{\log (z-\xi)\} \\
\boldsymbol{d} \psi^{(s)}(z) & =-k \int_{L} \overline{\gamma(s)} d\{\log (z-\xi)\}+\int_{L} \gamma(s) d\left\{\frac{\bar{\xi}}{z-\xi}\right\}
\end{aligned}
$$

where the density function $\gamma(\mathbf{s})$ is specified by (3). The complex potential functions (A.8) for the continuous distribution of line forces (or line dislocations) are called the single-layer potentials while those (A.9) for the force dipoles (or dislocation dipoles) double-layer potentials [28]. Further discussion on the layer potential functions are given by Denda and Dong [21]. Of interest to our present application are the single layer of line forces, (A.8) with $k=-\kappa$ and $\Gamma(s)=t(s) / 2 \pi(\kappa+1)$, and the double layer of dislocation dipoles, (A.9) with $k=1$ and $\gamma(s)=i \mu b(s) / \pi(\kappa+1)$, where $t(s)=t_{x}+i t_{y}$ is the traction and $b(s)=b_{x}+i b_{y}$ is the Burgers vector of the dislocation. 


\section{B Analytical Green's function for single crack}

\section{B.1 Image term solution}

Consider the line force $f$ (or the line dislocation $b$ ) at $\xi$ when a crack is present along the $x$-axis in the interval $(-a, a)$. The Green's function solution that satisfies the traction free crack surface condition is sought in the form

$$
\phi^{(t o t a l)}=\phi^{(s)}+\phi, \quad \psi^{(\text {total })}=\psi^{(s)}+\psi,
$$

where $\phi^{(s)}$ and $\psi^{(s)}$ are the singular parts (or singular terms) for the infinite homogeneous body and $\phi$ and $\psi$ are the regular parts (or image terms) to be determined so that the traction on the surface of the hole becomes zero. The singular terms are given in Appendix A. The image terms are determined with the help of the conformal mapping,

$$
z=M(w)=R\left(w+\frac{1}{w}\right) ; \quad R=\frac{a}{2}
$$

that maps the crack face into the unit circle and the points $z$ and $\xi$ in the $z$-plane into points $w$ and $\rho$ in the $w$-plane. They are given by (Denda and Kosaka [13]),

$$
\phi(w, \rho)=\phi_{0}(w, \rho) ; \quad \psi(w, \rho)=\psi_{0}(w, \rho)-z \frac{\phi_{0}^{\prime}(w, \rho)}{M^{\prime}(w)}
$$

where

$$
\begin{aligned}
\phi_{0}(w, \rho) & =\phi_{1}(w, \rho) \gamma+\phi_{2}(w, \rho) \bar{\gamma} \\
\psi_{0}(w, \rho) & =\psi_{1}(w, \rho) \gamma+\psi_{2}(w, \rho) \bar{\gamma}
\end{aligned}
$$

with

$$
\begin{aligned}
\phi_{1}(w, \rho) & =L\left(w, \frac{1}{\rho}\right)+k L\left(w, \frac{1}{\bar{\rho}}\right), \\
\phi_{2}(w, \rho) & =\frac{\bar{\xi}-\xi}{R\left(1-\overline{\rho^{2}}\right)} \frac{1}{w-\frac{1}{\bar{\rho}}}, \\
\psi_{1}(w, \rho) & =\frac{\xi-\bar{\xi}}{R\left(1-\rho^{2}\right)} \frac{1}{w-\frac{1}{\rho}}, \\
\psi_{2}(w, \rho) & =k L\left(w, \frac{1}{\rho}\right)+L\left(w, \frac{1}{\bar{\rho}}\right),
\end{aligned}
$$

and the function $L(w, \eta)$ is defined by

$$
L(w, \eta)=\ln (w-\eta)-\ln w,
$$

with $\eta=\frac{1}{\rho}$ or $\frac{1}{\bar{\rho}}$. The prime attached to the functions indicates the differentiation with respect to $w$. The constant $k$ and the coefficient $\gamma$ are defined by (3). 
The Green's function solutions for the displacement, traction and stress components are give in the form

$$
u^{(\text {total })}=u^{(s)}+u, \quad t^{(\text {total })}=t^{(s)}+t, \quad \sigma^{(\text {total })}=\sigma^{(s)}+\sigma, \quad \tau^{(\text {total })}=\tau^{(s)}+\tau,
$$

where $u^{(s)}, t^{(s)}, \sigma^{(s)}$ and $\tau^{(s)}$ are the contribution from the singular solution given in Section 2 and $u, t, \sigma$ and $\tau$ are the image terms derived below. Let $w$ and $\rho$ be the images of $z$ and $\xi$ by the mapping function $z=M(w)$ defined by (B.2). The displacement contribution from the image term is given by

$$
2 \mu u(w, \rho)=\kappa \phi_{0}(w, \rho)-\overline{\psi_{0}(w, \rho)}+(\bar{z}-z) \overline{\left(\frac{\phi_{0}^{\prime}(w, \rho)}{M^{\prime}(w)}\right)}
$$

and the stress contribution by

$$
\begin{aligned}
\sigma(w, \rho) & =2 \operatorname{Re}\left[\frac{\phi_{0}^{\prime}(w, \rho)}{M^{\prime}(w)}\right] \\
\tau(w, \rho) & =\frac{\psi_{0}^{\prime}(w, \rho)}{M^{\prime}(w)}-\frac{\phi_{0}^{\prime}(w, \rho)}{M^{\prime}(w)}+(\bar{z}-z) \frac{\phi_{0}^{\prime \prime}(w, \rho) M^{\prime}(w)-\phi_{0}^{\prime}(w, \rho) M^{\prime \prime}(w)}{\left(M^{\prime}(w)\right)^{3}}
\end{aligned}
$$

where the functions $\phi_{0}(w, \rho)$ and $\psi_{0}(w, \rho)$ are defined by (B.4) and $\sigma$ and $\tau$ by (A.2). The prime attached to the functions indicates the differentiation with respect to $w$. The traction on a line segment with the unit normal $n_{x}+i n_{y}=e^{i \alpha}$ is given by

$$
t(w, \rho)=e^{i \alpha} \sigma(w, \rho)-e^{-i \alpha} \overline{\tau(w, \rho)} .
$$

\section{B.2 Crack opening displacement}

The crack opening displacement $\delta u=u^{+}-u^{-}$is given, from (B.8) and Appendix B, by

$$
\begin{aligned}
2 \mu \delta u & \left.=\left\{\kappa\left[\delta L\left(w, \frac{1}{\rho}\right)+k \delta L\left(w, \frac{1}{\bar{\rho}}\right)\right]-\overline{\delta L\left(w, \frac{1}{\bar{\rho}}\right)}+k \overline{\delta L\left(w, \frac{1}{\rho}\right)}\right]\right\} \gamma \\
& +(\kappa+1) \frac{\bar{\xi}-\xi}{R\left(1-\bar{\rho}^{2}\right)} \delta\left(\frac{1}{w-\frac{1}{\bar{\rho}}}\right) \bar{\gamma}
\end{aligned}
$$

where

$$
\begin{aligned}
& \delta L\left(w, \frac{1}{\rho}\right)=L^{+}\left(w, \frac{1}{\rho}\right)-L^{-}\left(w, \frac{1}{\rho}\right), \\
& \delta L\left(w, \frac{1}{\bar{\rho}}\right)=L^{+}\left(w, \frac{1}{\bar{\rho}}\right)-L^{-}\left(w, \frac{1}{\bar{\rho}}\right),
\end{aligned}
$$

and the function $L(w, \eta)$ for $\eta=1 / \rho, 1 / \bar{\rho}$ is defined by (B.6). The constants $\gamma$ and $k$ are given by (A.4) for the line force and dislocation. The argument $w$ takes the values

$$
w^{ \pm}=\frac{x \pm i \sqrt{a+x} \sqrt{a-x}}{a}
$$

on the upper $(+)$ and lower $(-)$ faces of the crack. 


\section{B.3 Stress Intensity Factor}

The stress intensity factor is obtained using the stress formula (B.9) along the crack line with the result

$\left(K_{I}+i K_{I I}\right)( \pm a)=2 \sqrt{\frac{\pi}{a}}\left\{\frac{\bar{\xi}-\xi}{R\left(1-\rho^{2}\right)} \frac{1}{\left( \pm 1-\frac{1}{\rho}\right)^{2}} \gamma+\left[k\left(\frac{1}{ \pm 1-\frac{1}{\rho}} \mp 1\right)+\left(\frac{1}{ \pm 1-\frac{1}{\bar{\rho}}} \mp 1\right)\right] \bar{\gamma}\right\}$

where the sign \pm and $\mp$ follows the location $\pm a$ of the crack tips.

\section{Cauchy-type integrals}

Consider the Chebyshev polynomials, $T_{m}(x)$ and $U_{m-1}(x)$, of the first and second kind and define, as in [29], two Cauchy-type integrals

$$
\begin{aligned}
T^{(m)}(z) & =-\frac{1}{\pi} \int_{-1}^{1} \frac{\sqrt{1-x^{2}} U_{m-1}(x) d x}{x-z} \quad(m \geq 0), \\
U^{(m-1)}(z) & =\frac{1}{\pi} \int_{-1}^{1} \frac{T_{m}(x) d x}{\sqrt{1-x^{2}}(x-z)} \quad(m \geq 0)
\end{aligned}
$$

where $z=x+i y$ is a complex variable. These integrals are evaluated analytically, using the Cauchy integral formula, with the result

$$
\begin{aligned}
T^{(m)}(z) & =\left(z-\sqrt{z^{2}-1}\right)^{m} \quad(m \geq 0), \\
U^{(m-1)}(z) & =-\frac{\left(z-\sqrt{z^{2}-1}\right)^{m}}{\sqrt{z^{2}-1}} \quad(m \geq 0) .
\end{aligned}
$$

Note that $T^{(0)}(z)=1$ and $U^{(-1)}(z)=-1 / \sqrt{z^{2}-1}$. 


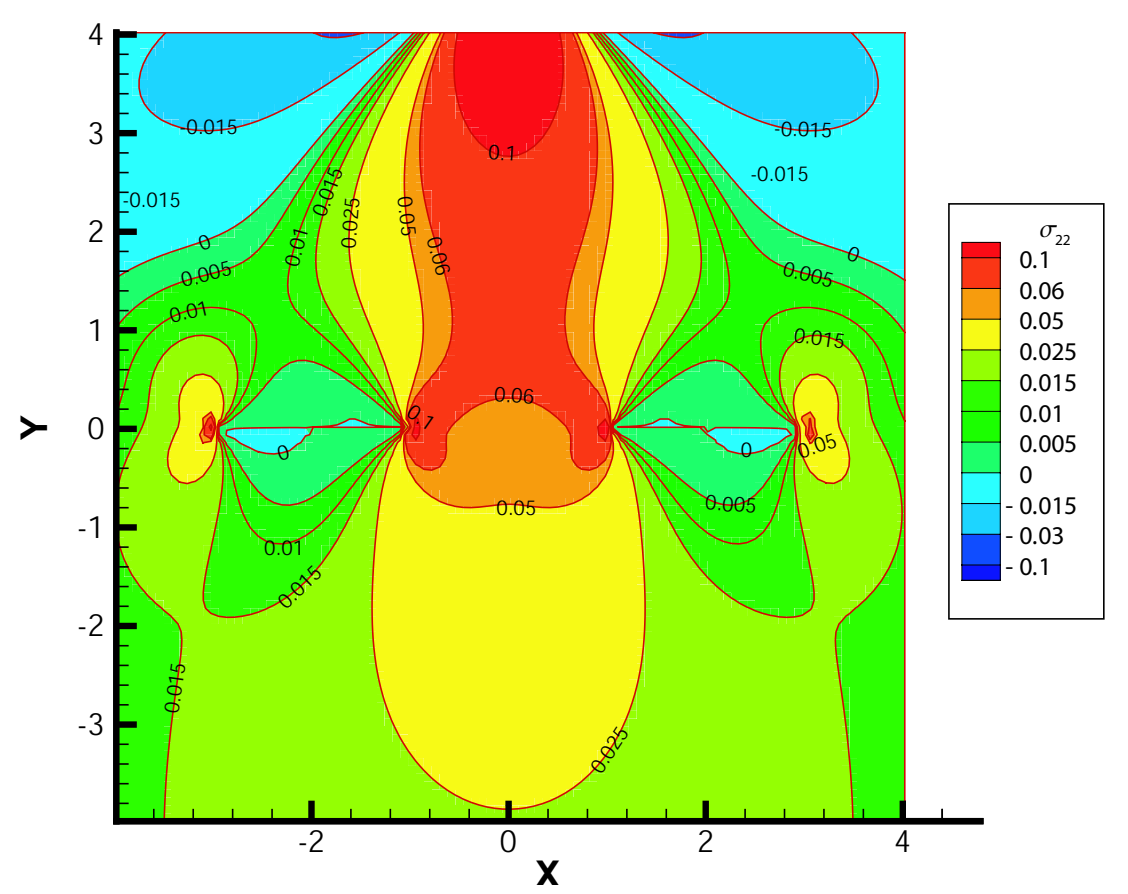

(a)

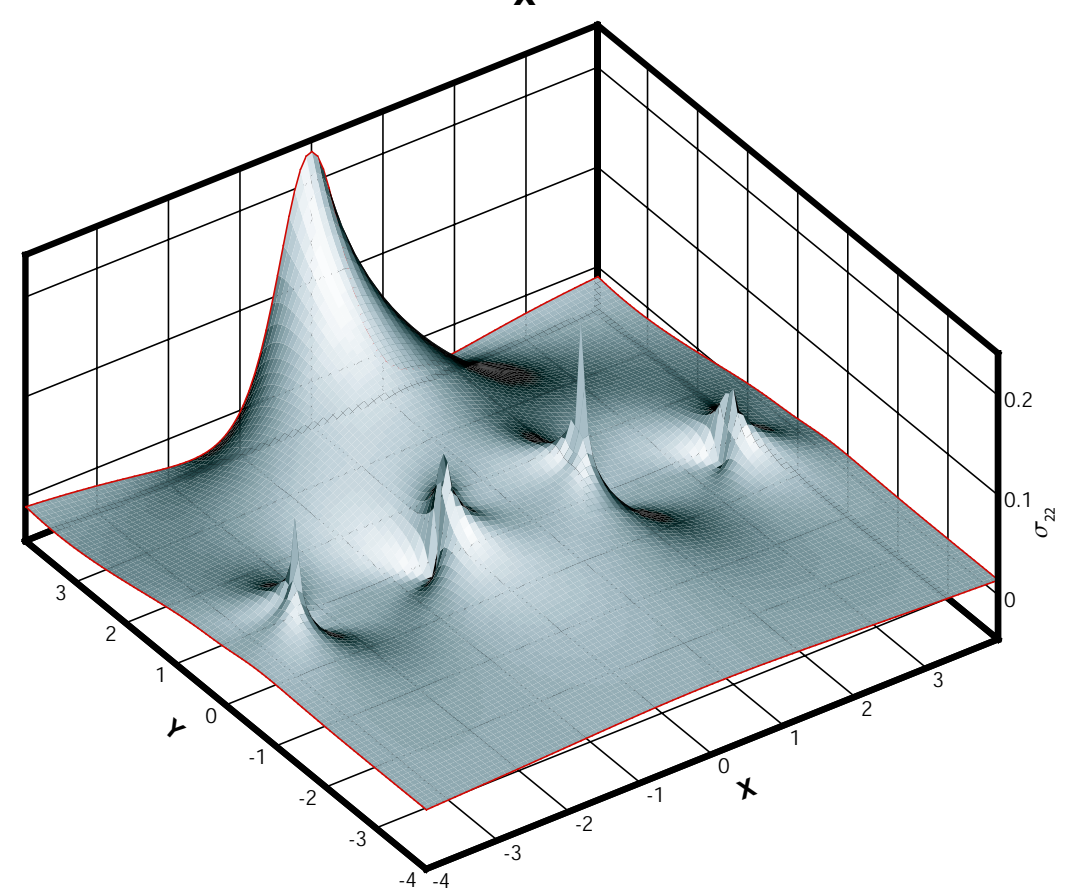

(b)

Figure 10: Variation of $\sigma_{y y}$ by $b_{x}=1$ as a function of location in infinite region with two co-linear cracks for (a) 2-D color contour and (b) 3-D shaded plot. 


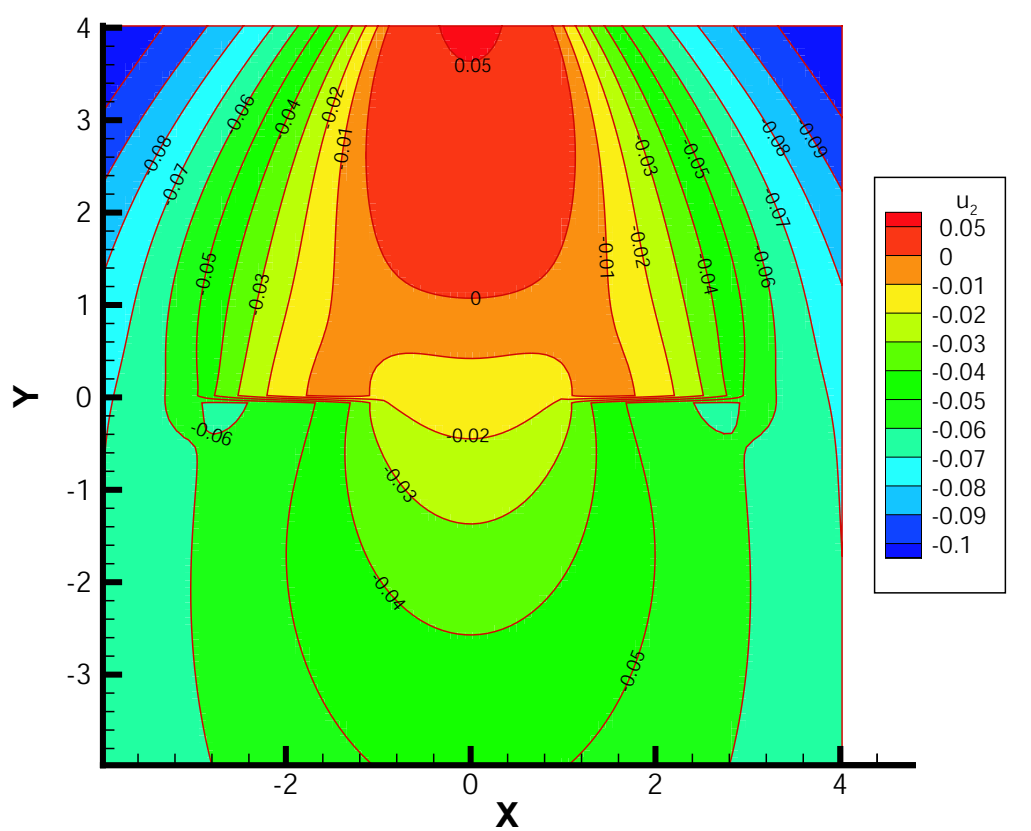

(a)

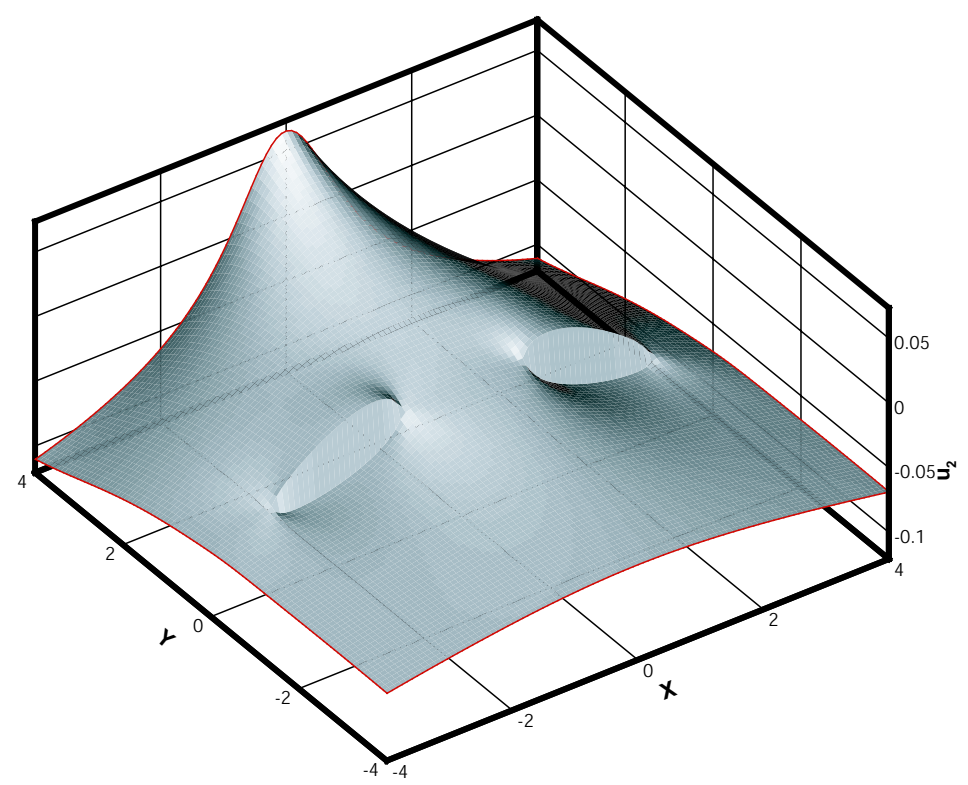

(b)

Figure 11: Variation of $u_{y}$ by $b_{x}=1$ as a function of location in infinite region with two co-linear cracks for (a) 2-D color contour and (b) 3-D shaded plot. 


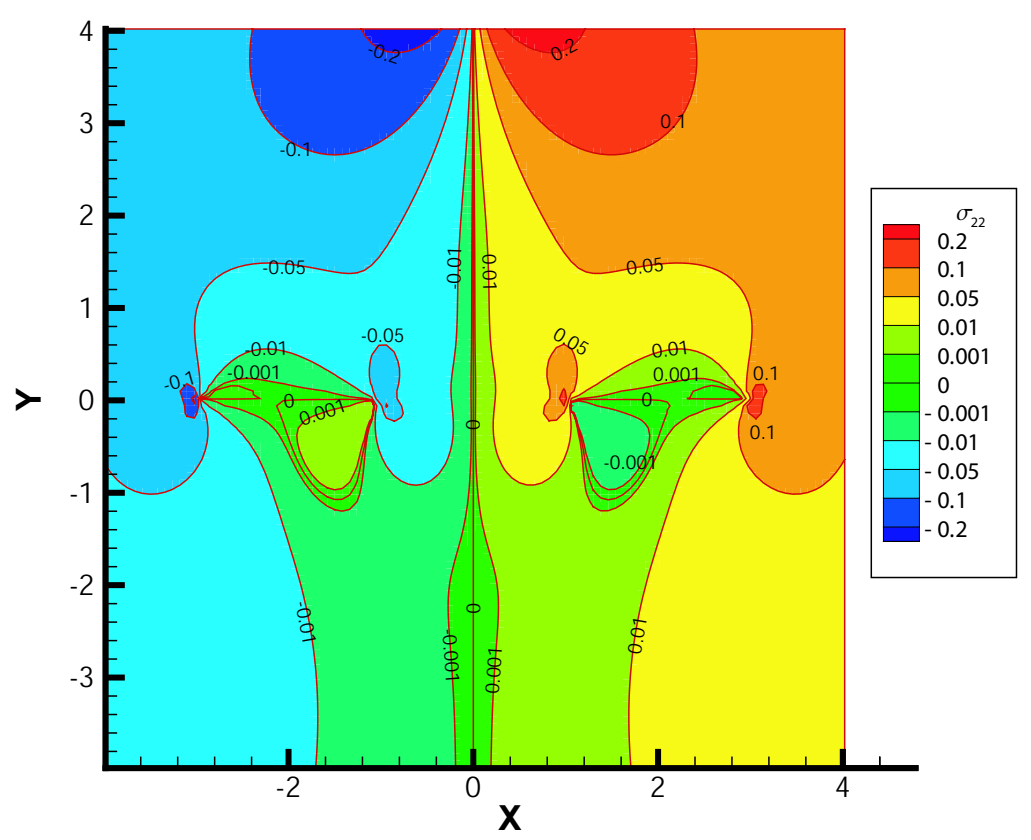

(a)

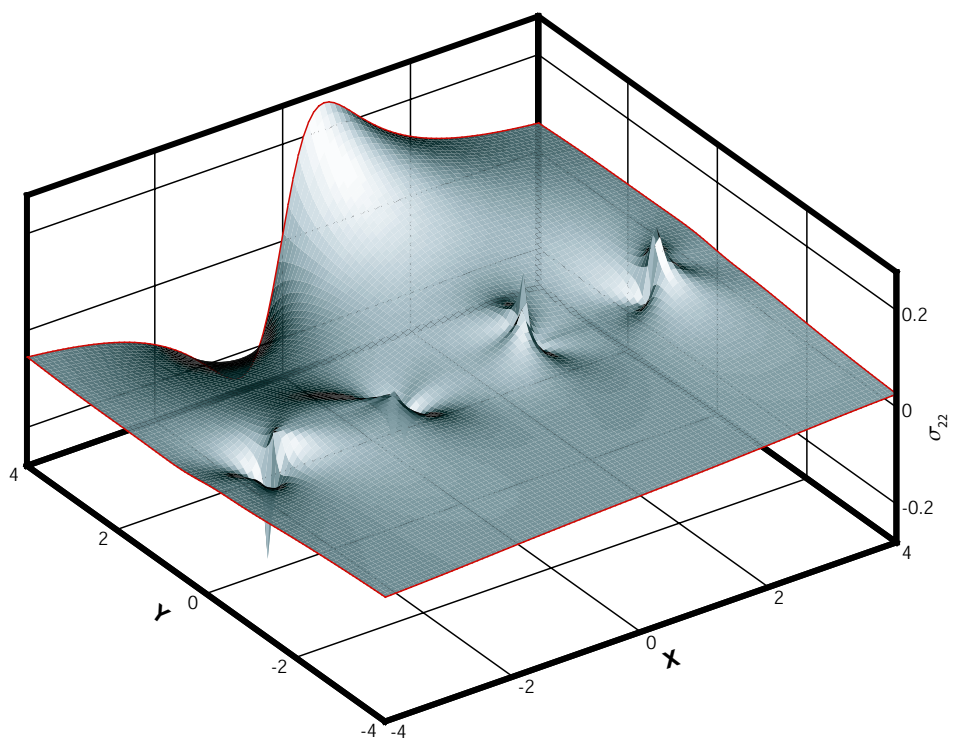

(b)

Figure 12: Variation of $\sigma_{y y}$ by $b_{y}=1$ as a function of location in infinite region with two co-linear cracks for (a) 2-D color contour and (b) 3-D shaded plot. 


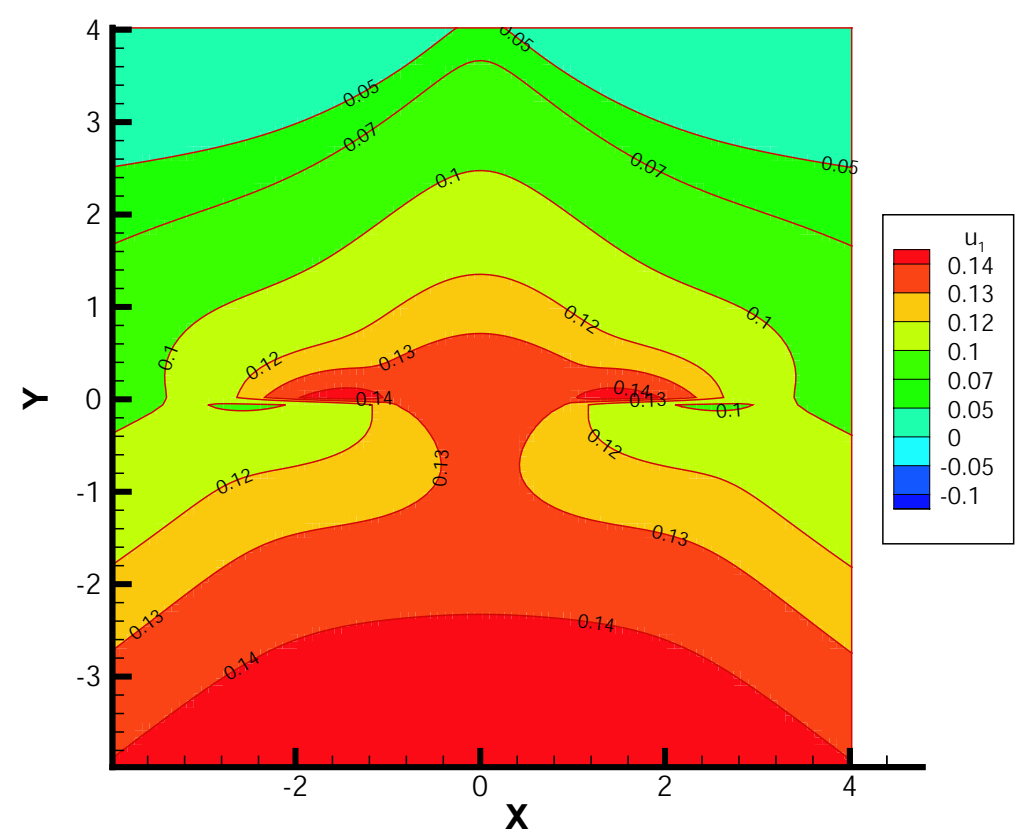

(a)

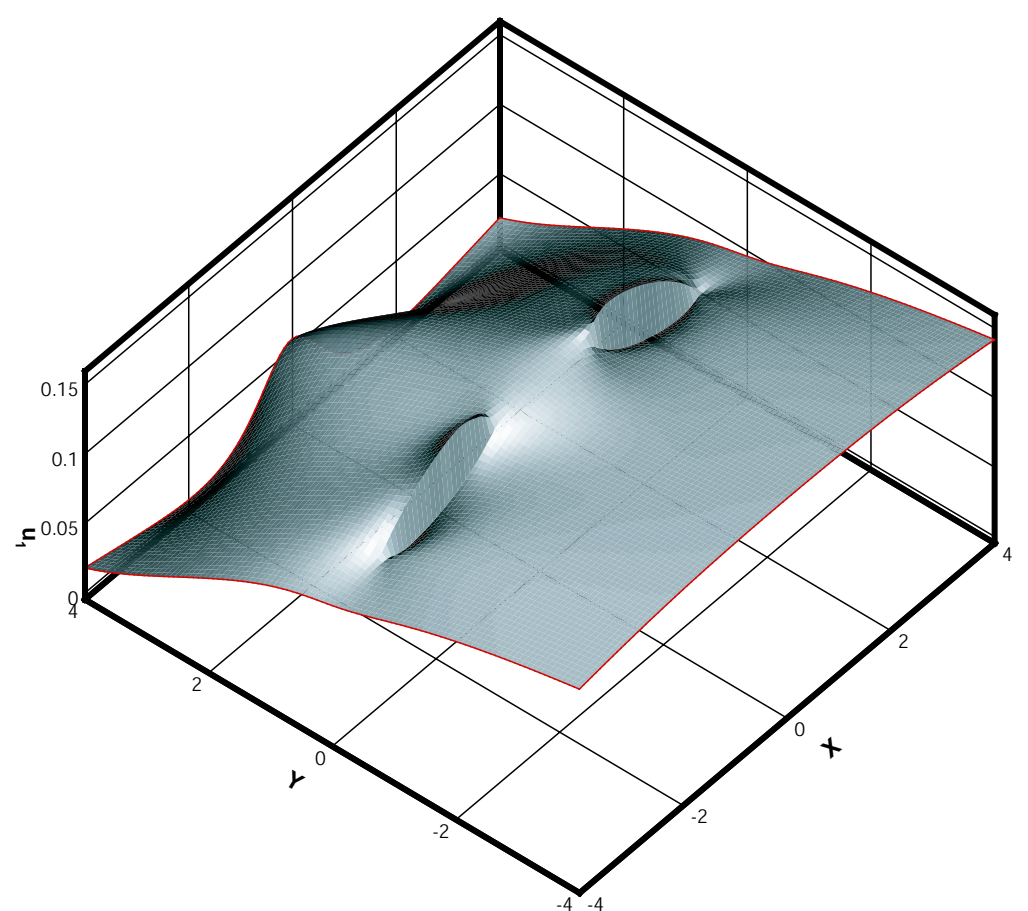

(b)

Figure 13: Variation of $u_{x}$ by $b_{y}=1$ as a function of location in infinite region with two co-linear cracks for (a) 2-D color contour and (b) 3-D shaded plot. 


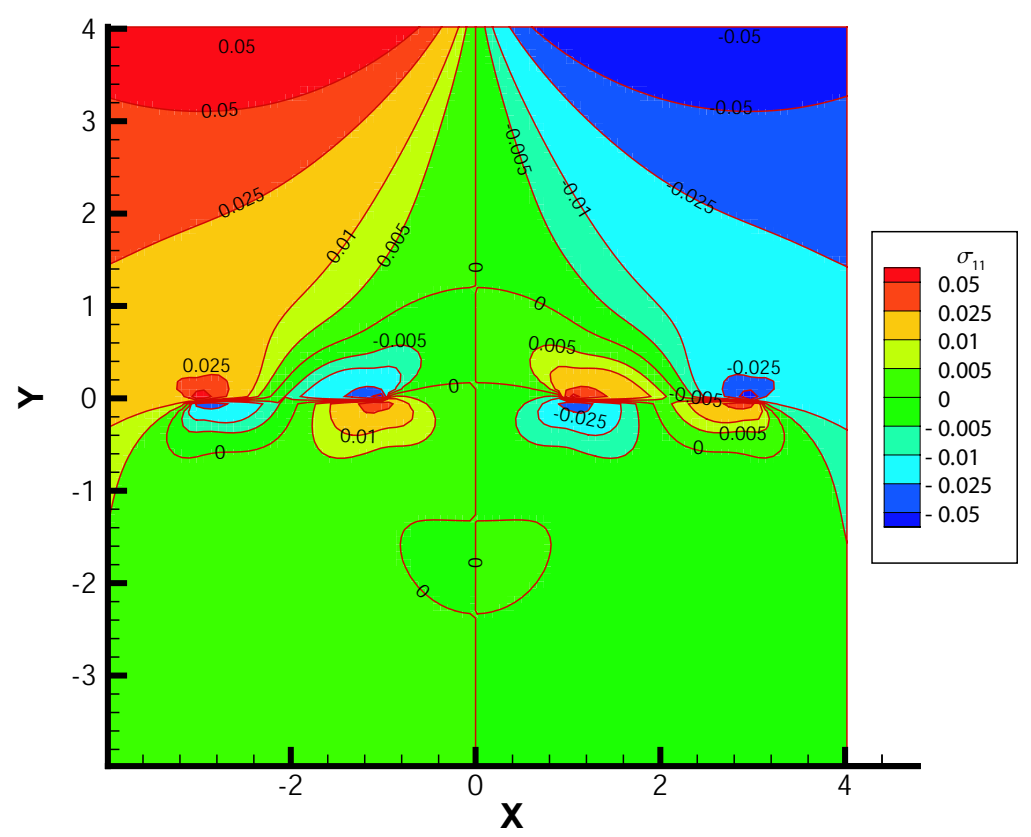

(a)

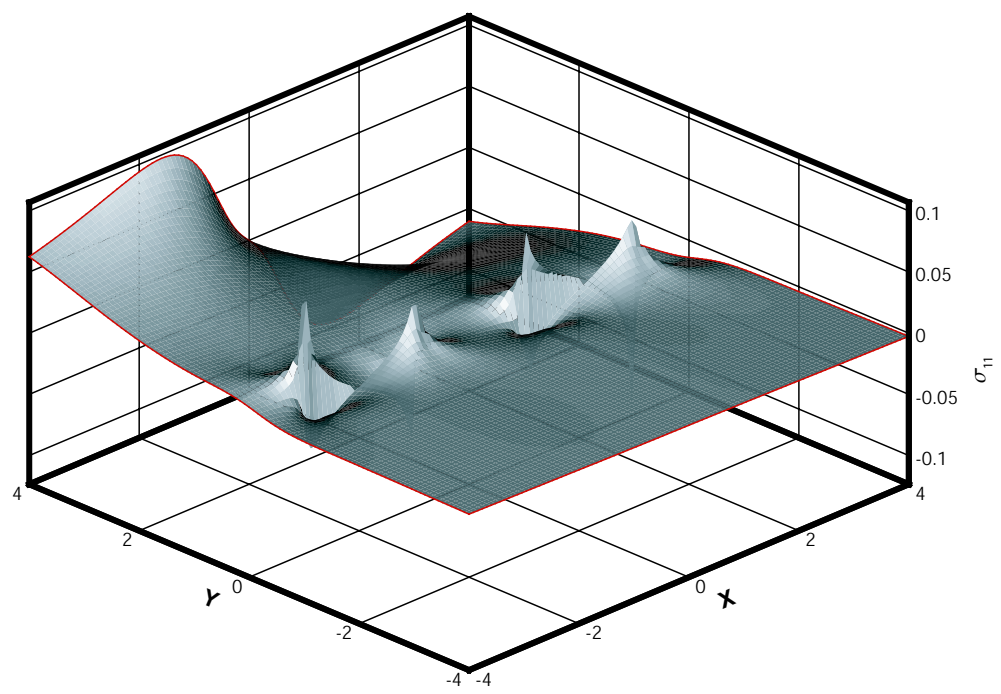

(b)

Figure 14: Variation of $\sigma_{x x}$ by $f_{x}=1$ as a function of location in infinite region with two co-linear cracks for (a) 2-D color contour and (b) 3-D shaded plot. 

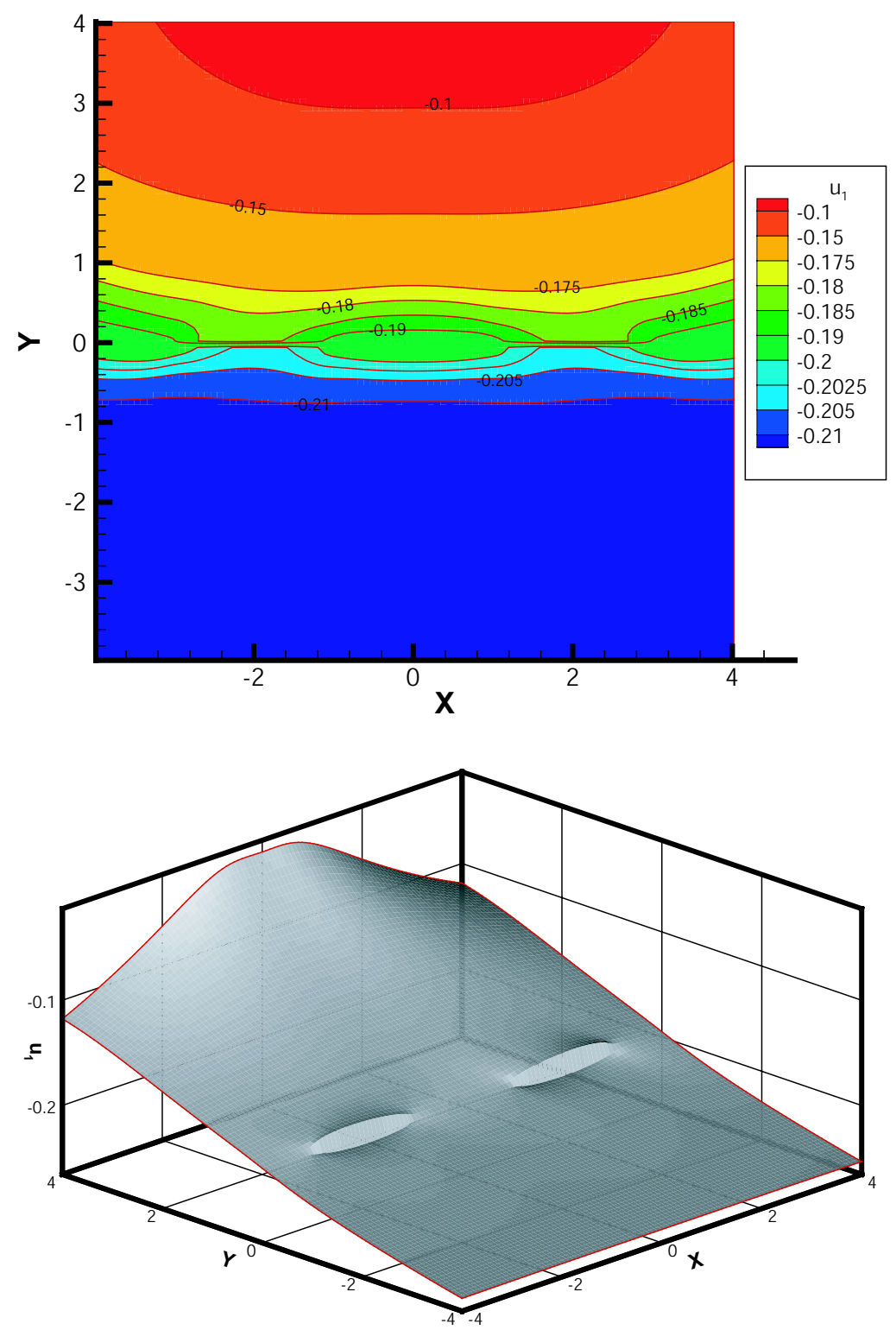

(b)

Figure 15: Variation of $u_{x}$ by $f_{x}=1$ as a function of location in infinite region with two co-linear cracks for (a) 2-D color contour and (b) 3-D shaded plot. 

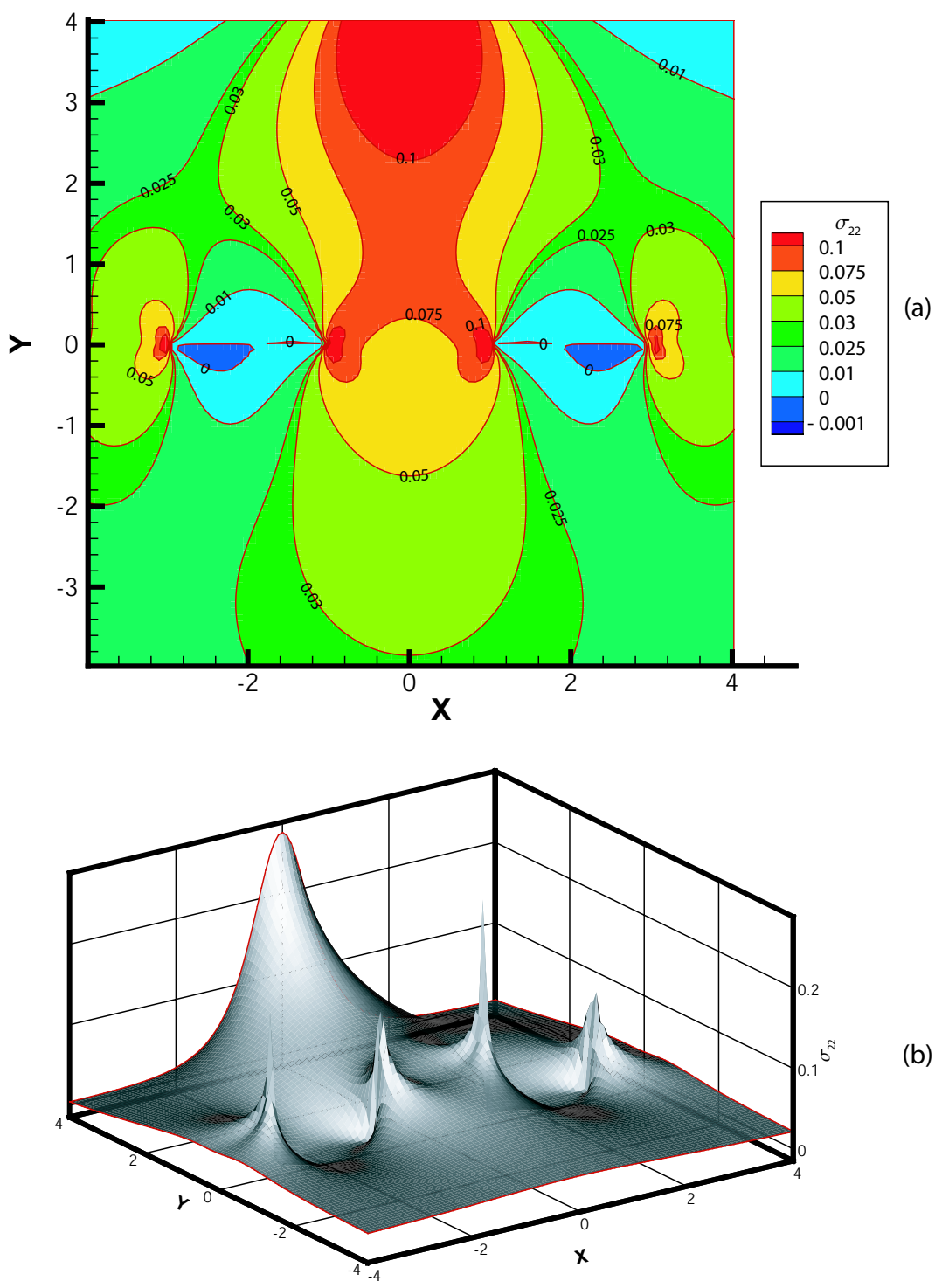

Figure 16: Variation of $\sigma_{y y}$ by $f_{y}=1$ as a function of location in infinite region with two co-linear cracks for (a) 2-D color contour and (b) 3-D shaded plot. 

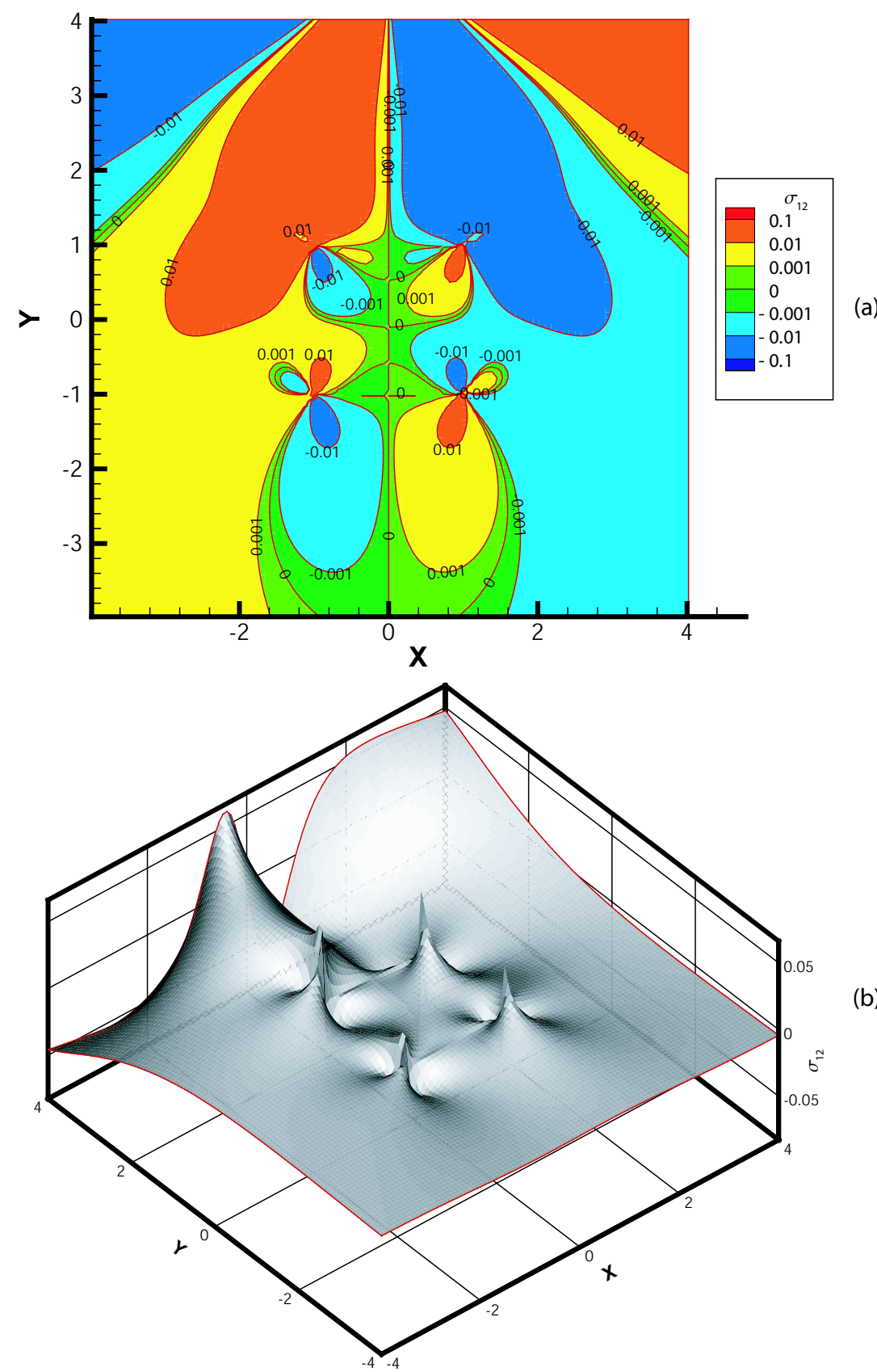

Figure 17: Variation of $\sigma_{x y}$ by $b_{x}=1$ as a function of location in infinite region with two parallel cracks for (a) 2-D color contour and (b) 3-D shaded plot. 


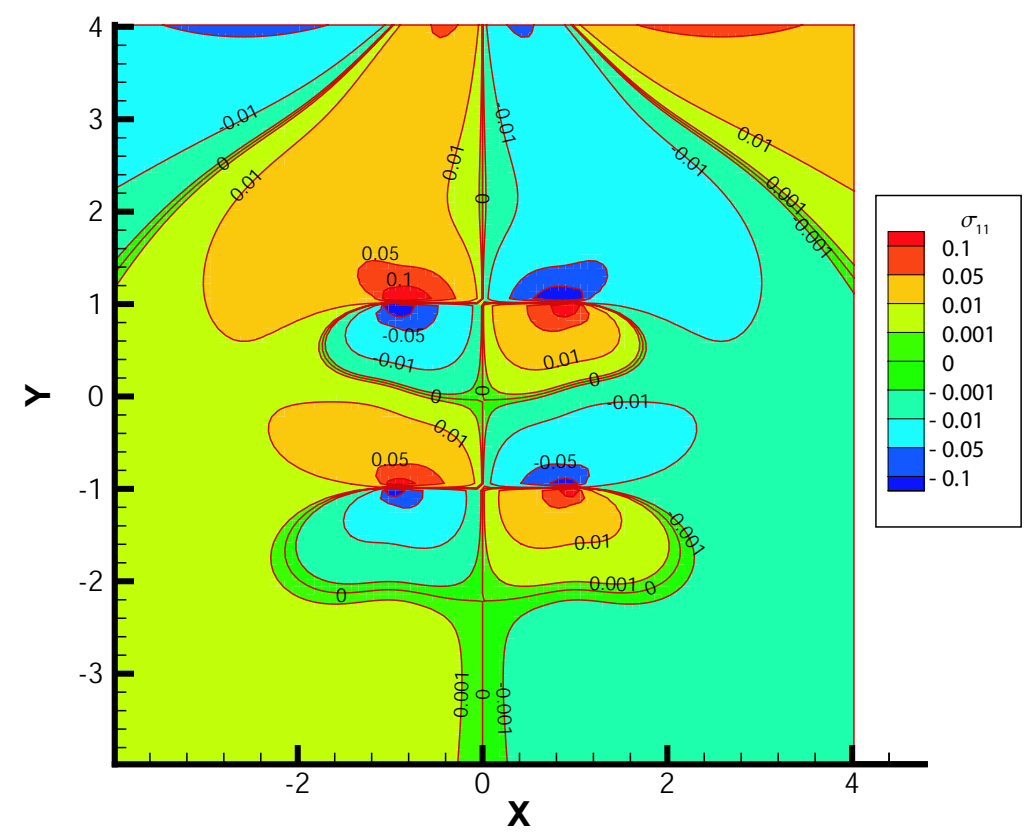

(a)

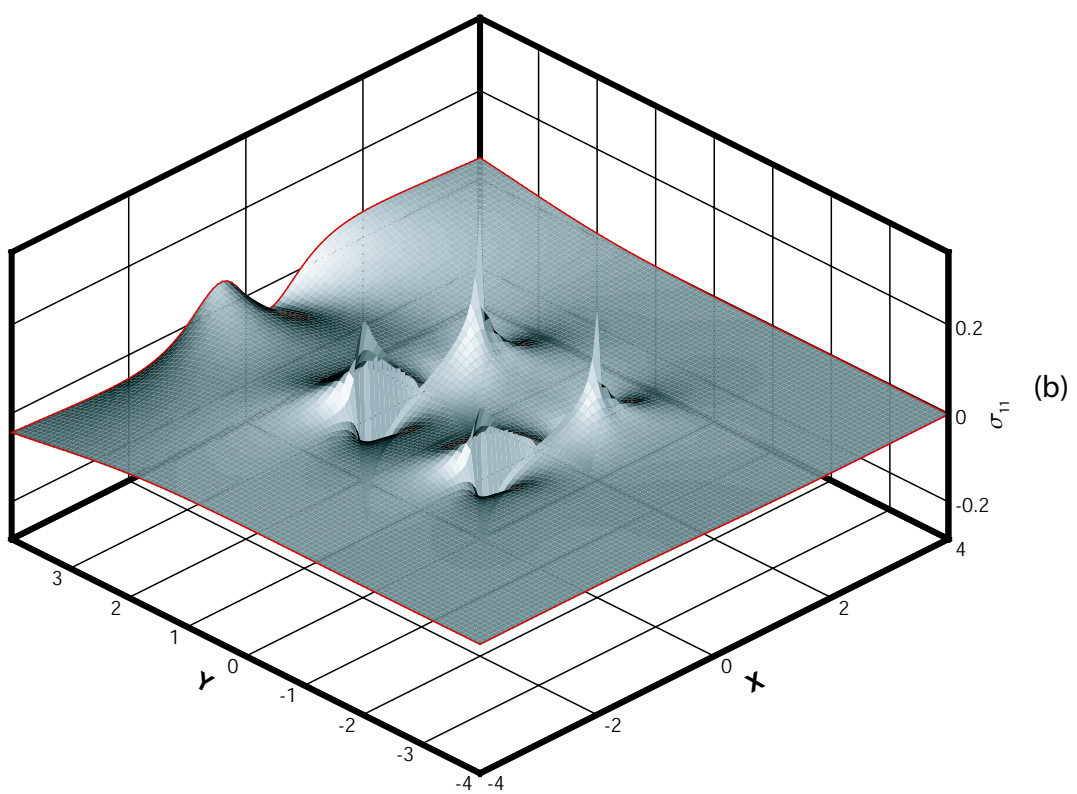

Figure 18: Variation of $\sigma_{x x}$ by $b_{y}=1$ as a function of location in infinite region with two parallel cracks for (a) 2-D color contour and (b) 3-D shaded plot. 


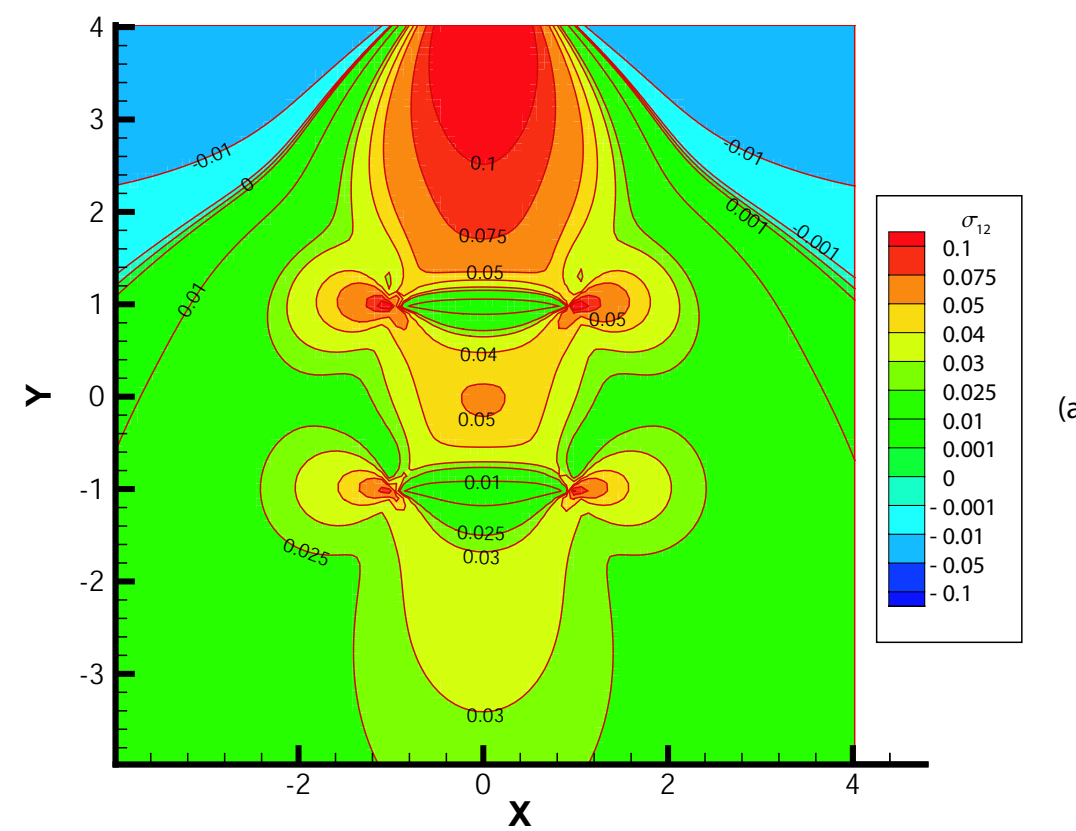

(a)

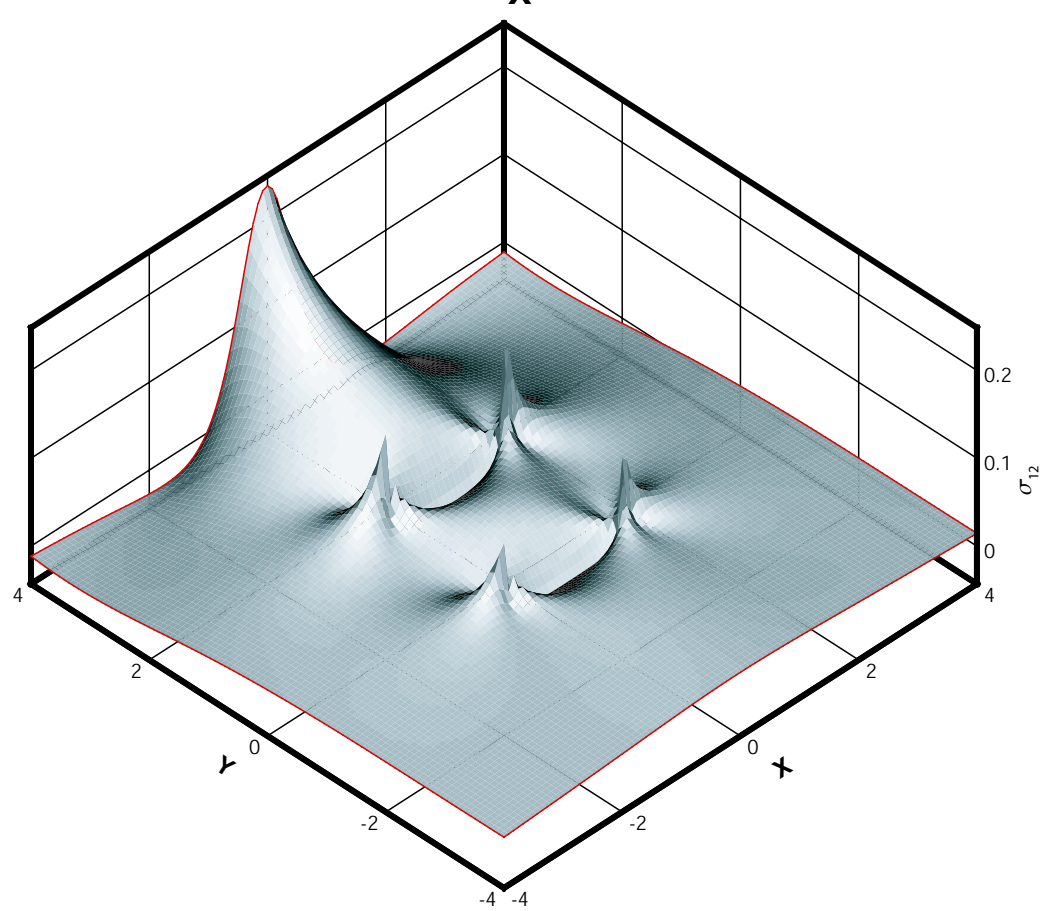

(b)

Figure 19: Variation of $\sigma_{x y}$ by $b_{y}=1$ as a function of location in infinite region with two parallel cracks for (a) 2-D color contour and (b) 3-D shaded plot. 

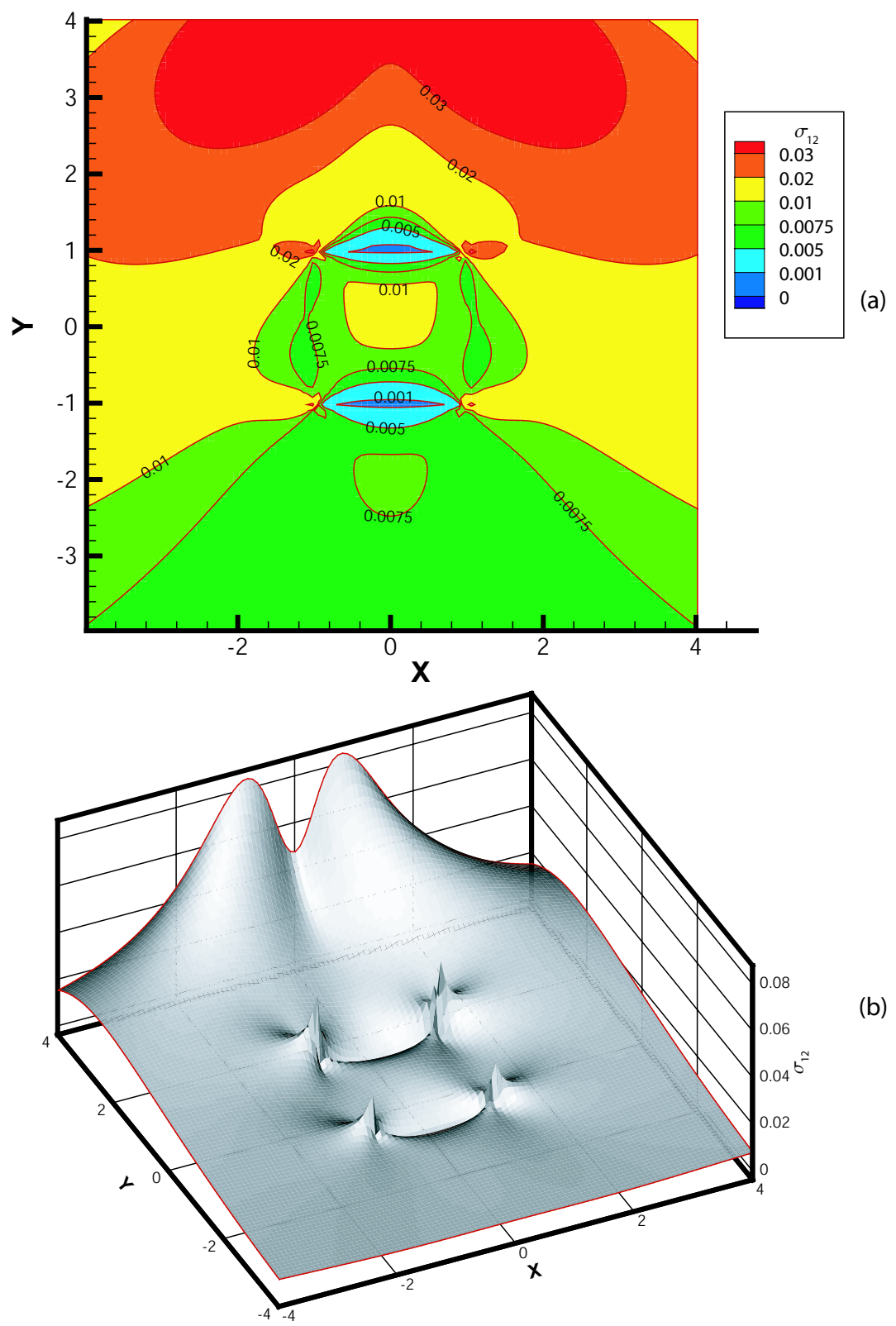

Figure 20: Variation of $\sigma_{x y}$ by $f_{x}=1$ as a function of location in infinite region with two parallel cracks for (a) 2-D color contour and (b) 3-D shaded plot. 


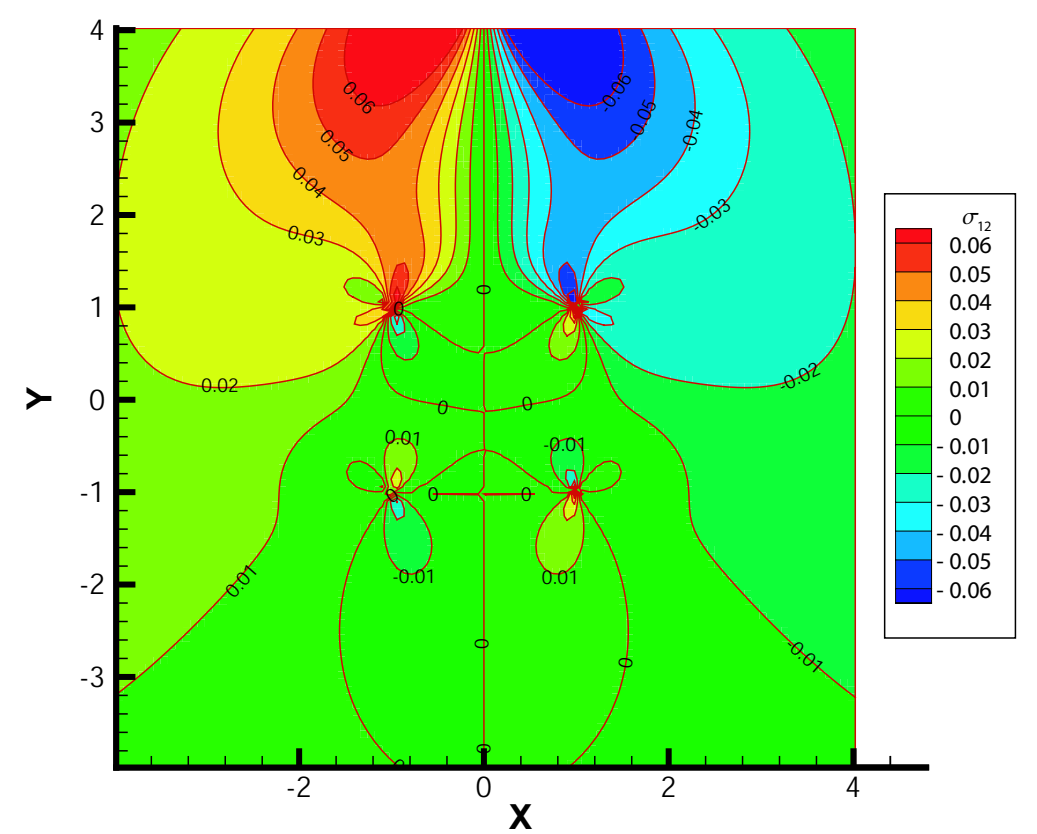

(a)

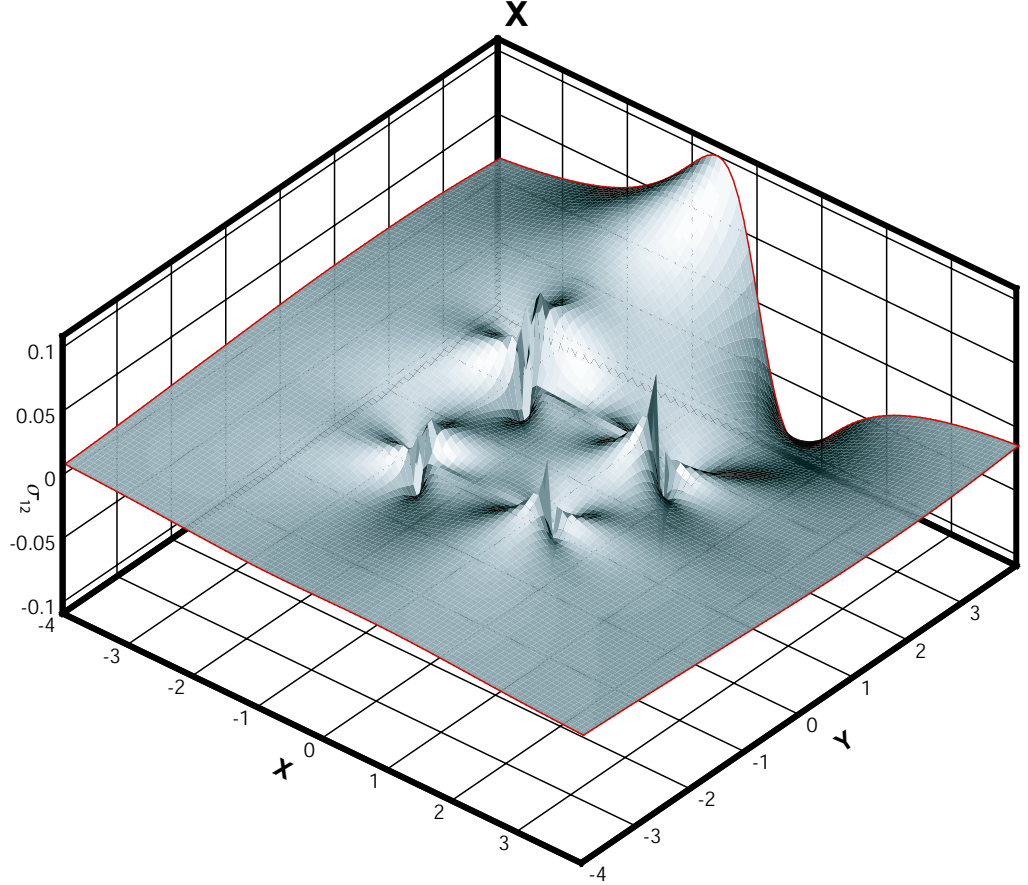

(b)

Figure 21: Variation of $\sigma_{x y}$ by $f_{y}=1$ as a function of location in infinite region with two parallel cracks for (a) 2-D color contour and (b) 3-D shaded plot. 


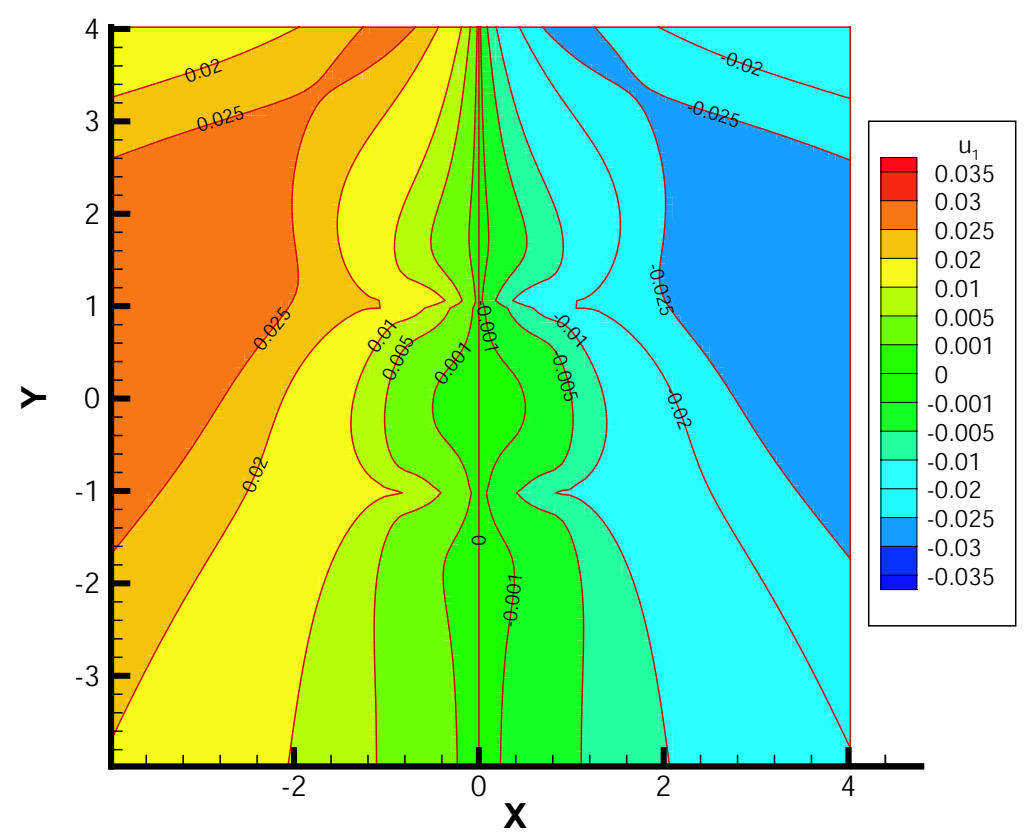

(a)

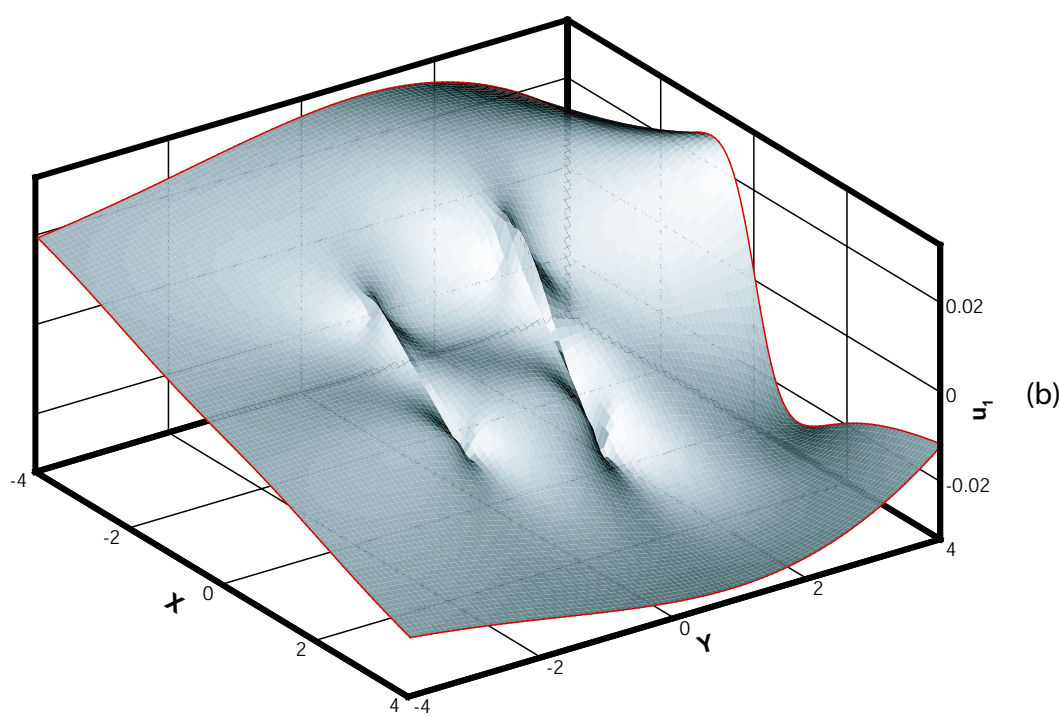

Figure 22: Variation of $u_{x}$ by $f_{y}=1$ as a function of location in infinite region with two parallel cracks for (a) 2-D color contour and (b) 3-D shaded plot. 


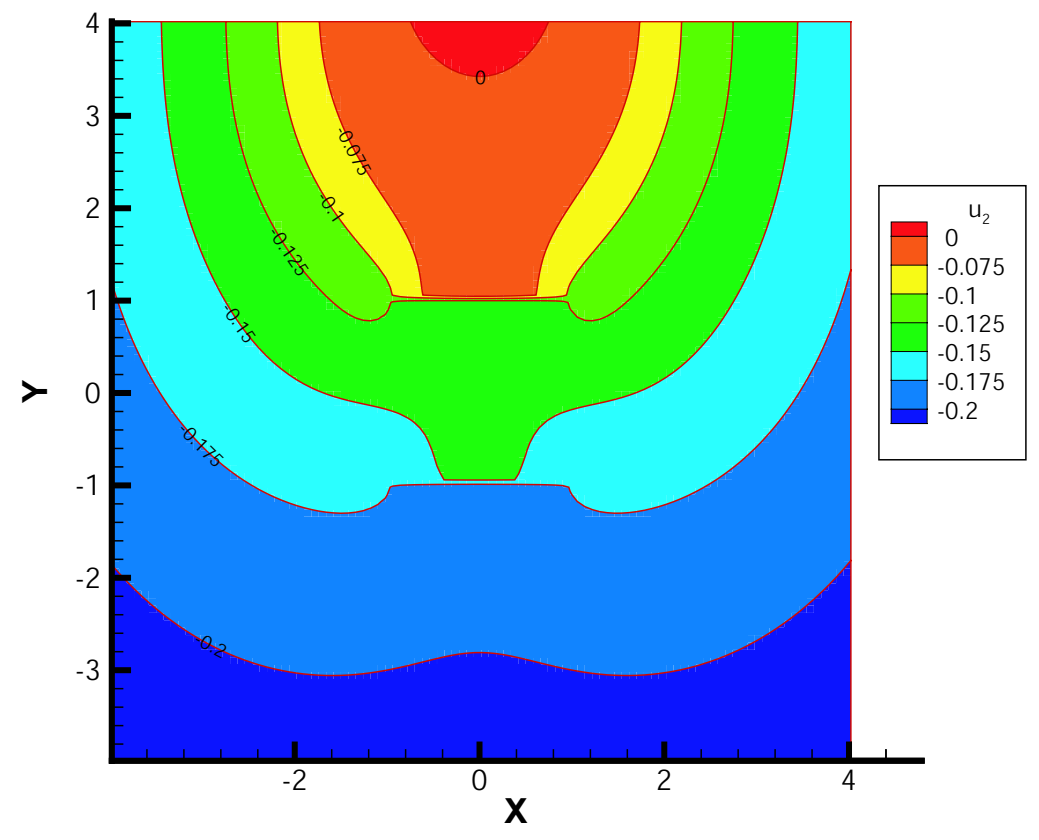

(a)

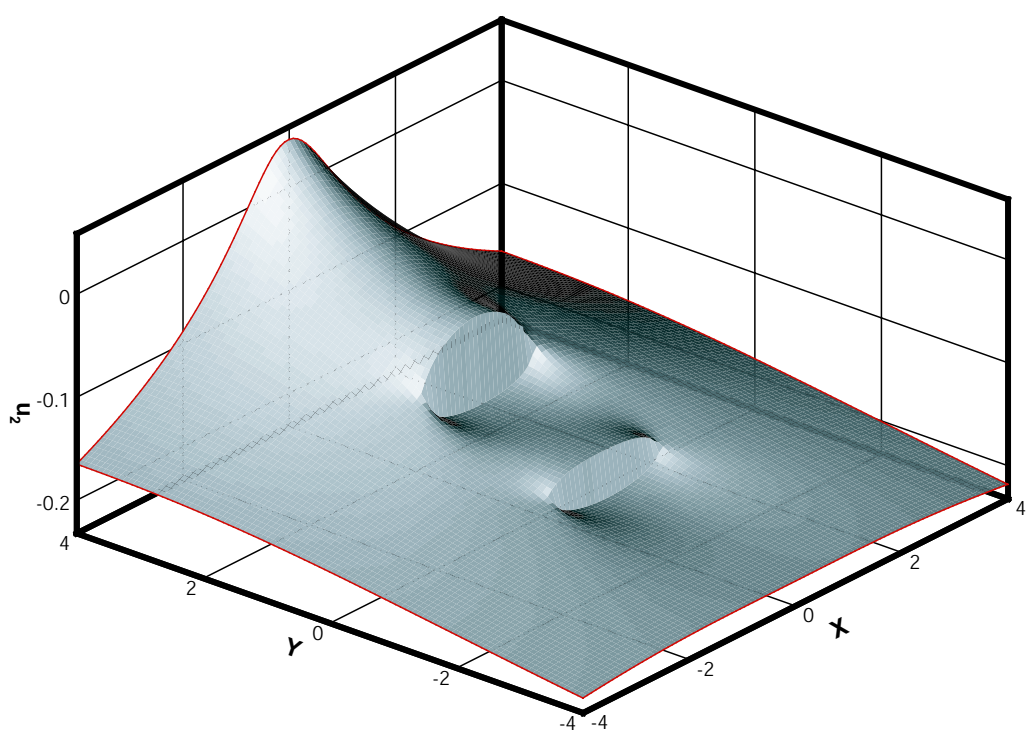

(b)

Figure 23: Variation of $u_{y}$ by $f_{y}=1$ as a function of location in infinite region with two parallel cracks for (a) 2-D color contour and (b) 3-D shaded plot. 


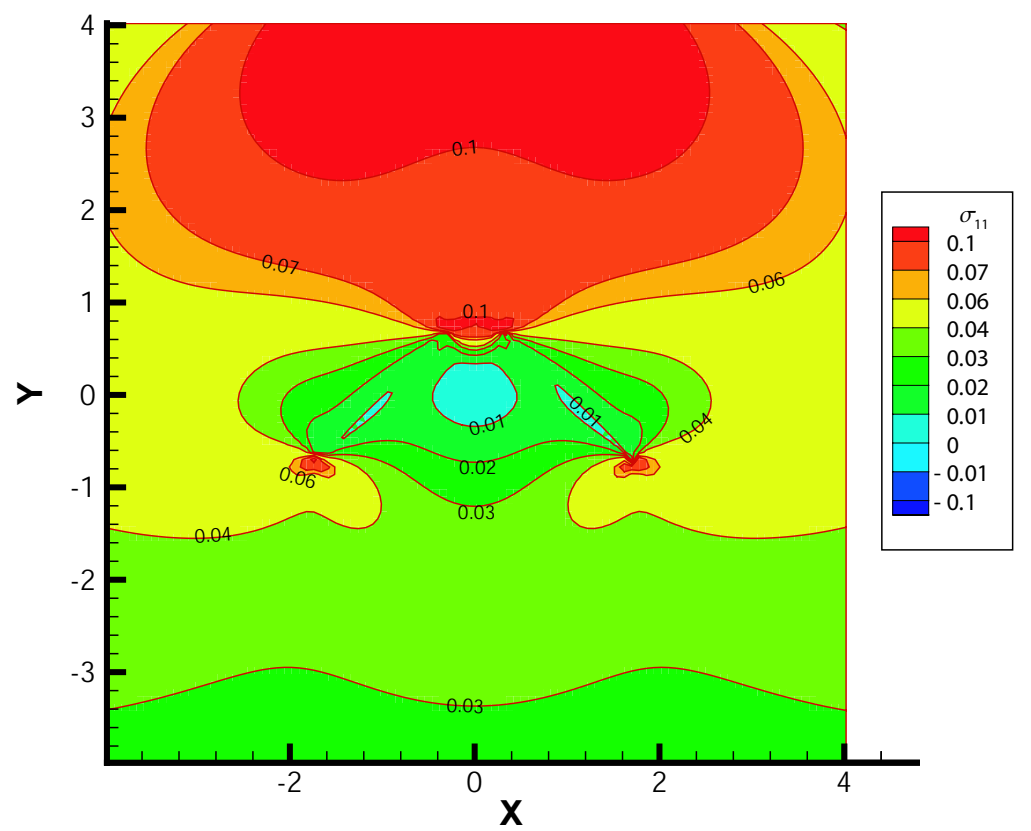

(a)

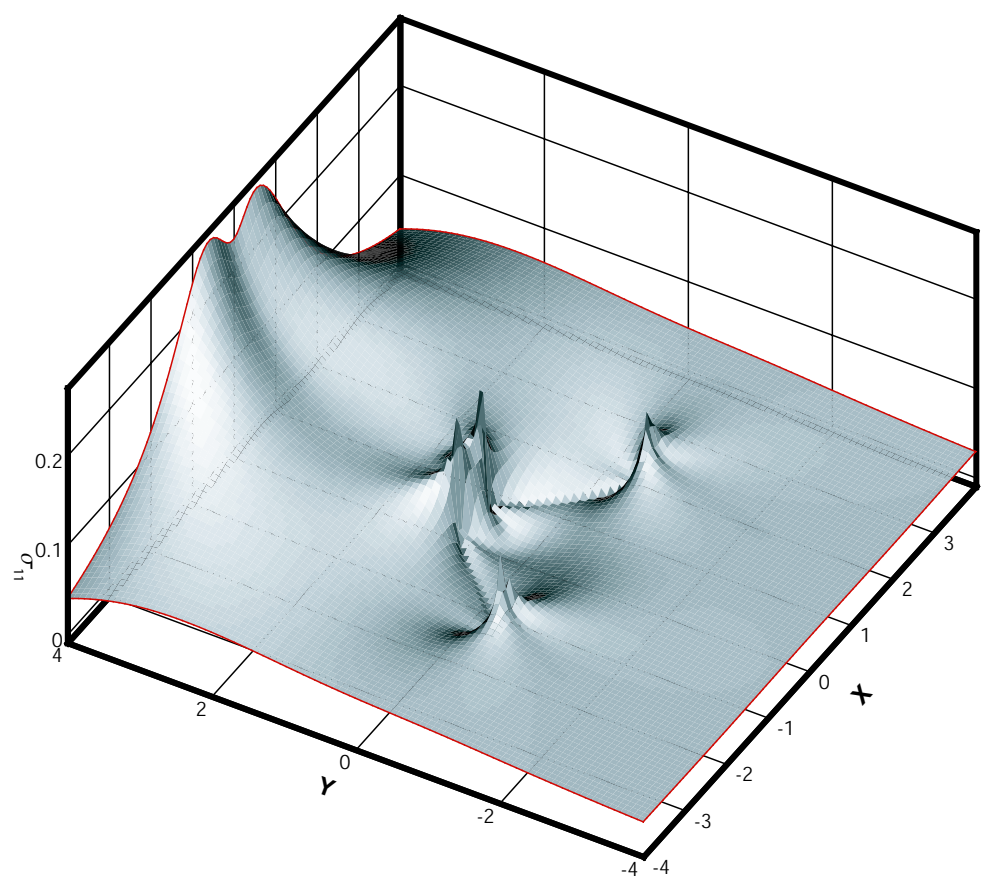

(b)

Figure 24: Variation of $\sigma_{x x}$ by $b_{x}=1$ as a function of location in infinite region with two inclined cracks for (a) 2-D color contour and (b) 3-D shaded plot. 


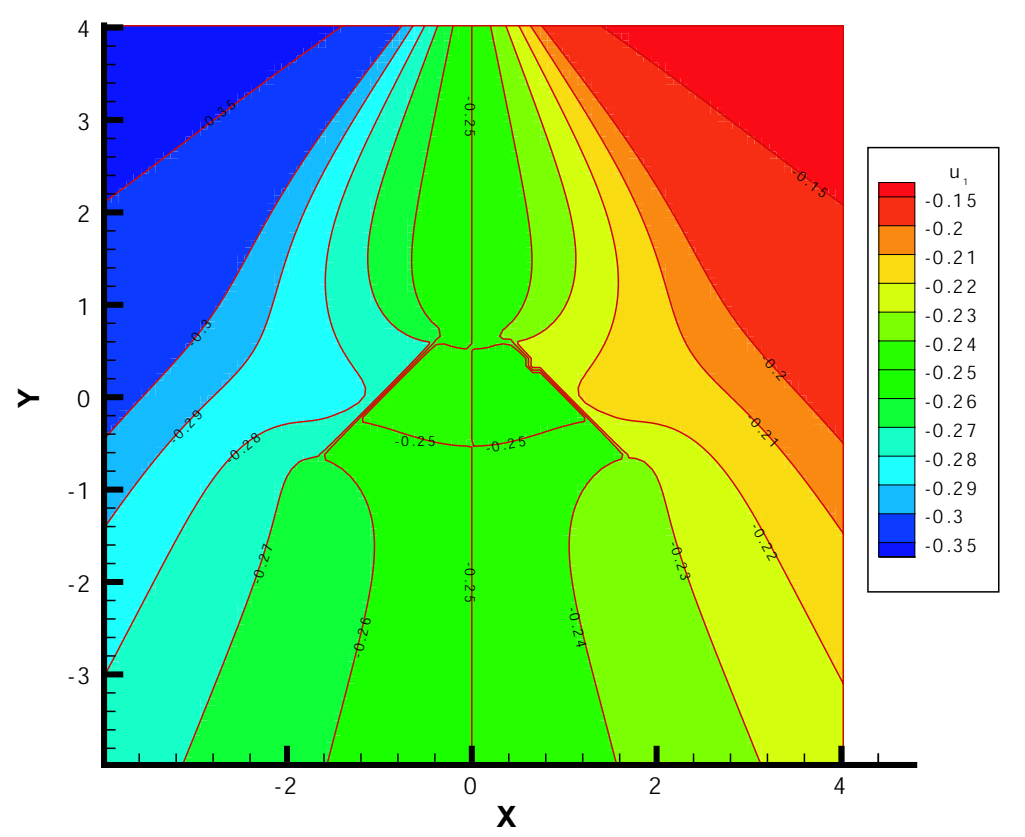

(a)

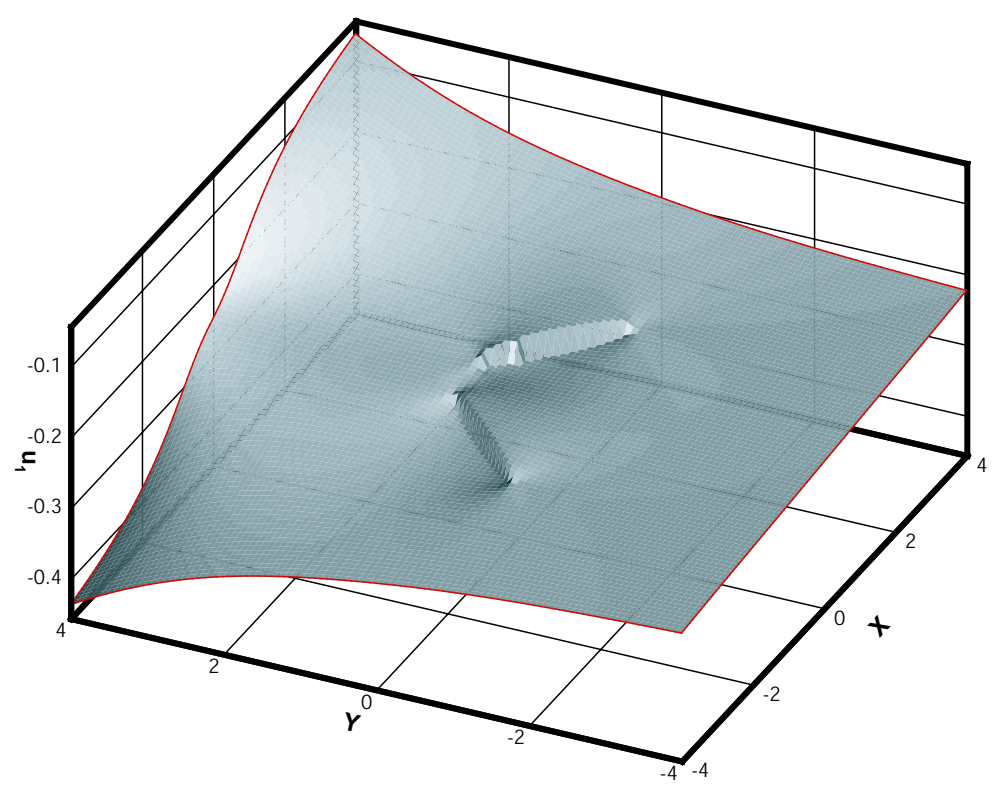

(b)

Figure 25: Variation of $u_{x}$ by $b_{x}=1$ as a function of location in infinite region with two inclined cracks for (a) 2-D color contour and (b) 3-D shaded plot. 


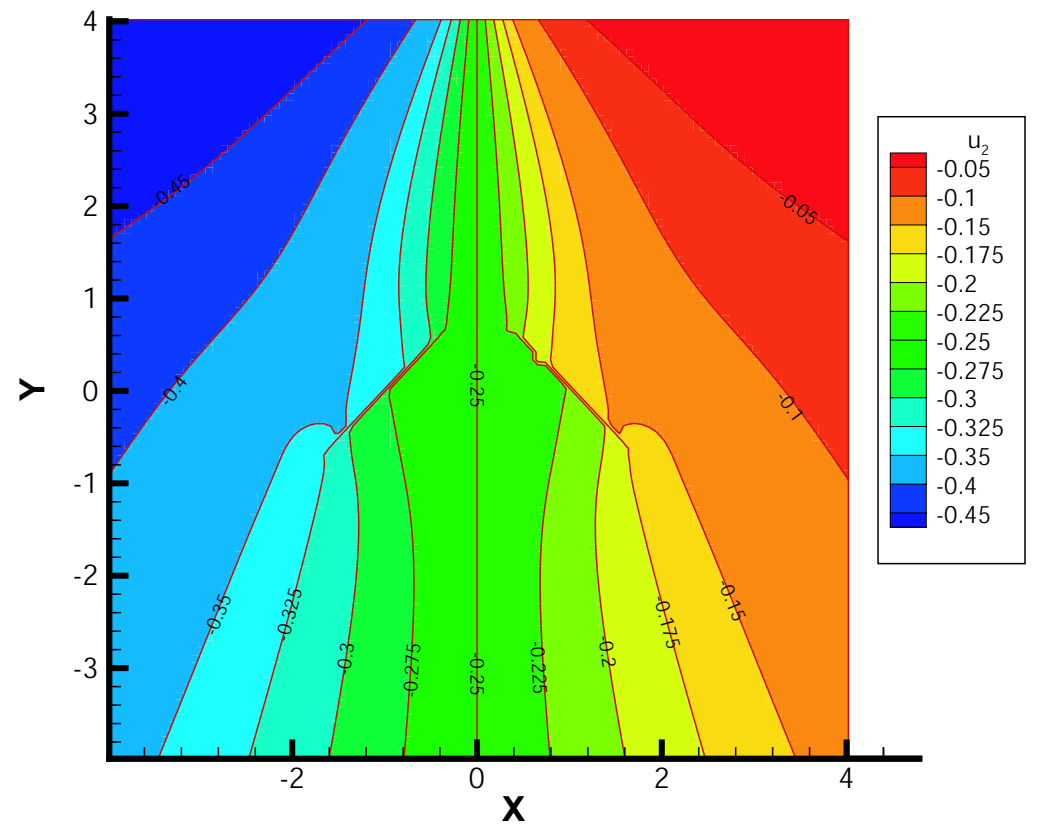

(a)

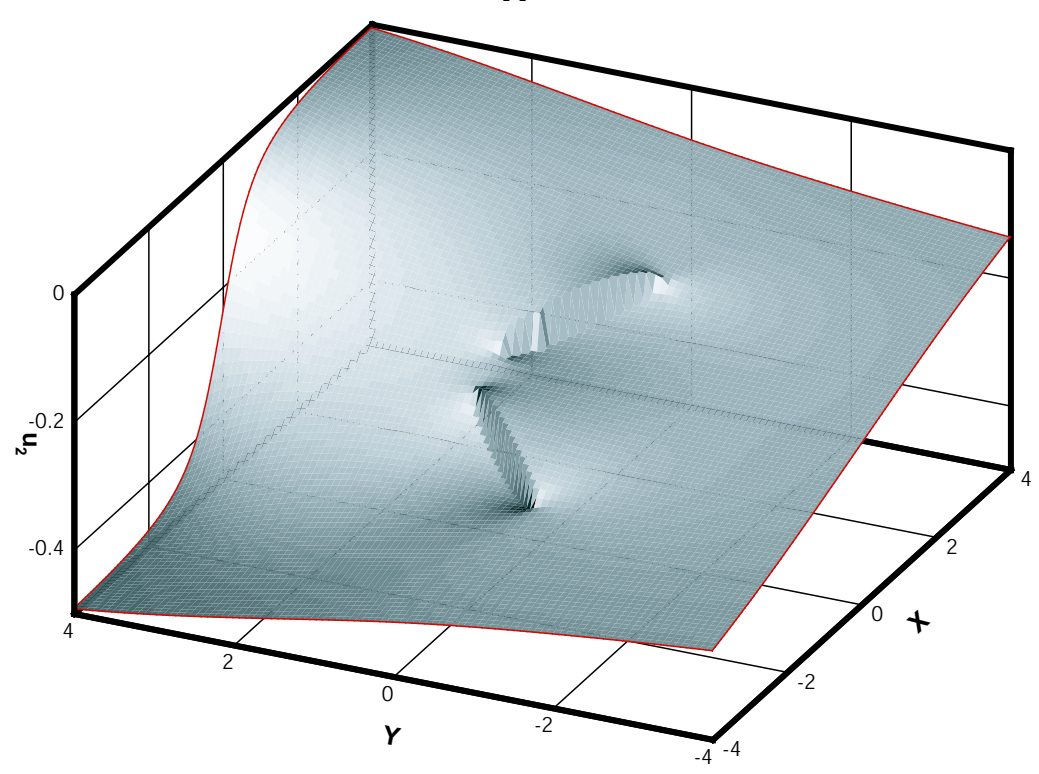

(b)

Figure 26: Variation of $u_{y}$ by $b_{y}=1$ as a function of location in infinite region with two inclined cracks for (a) 2-D color contour and (b) 3-D shaded plot. 


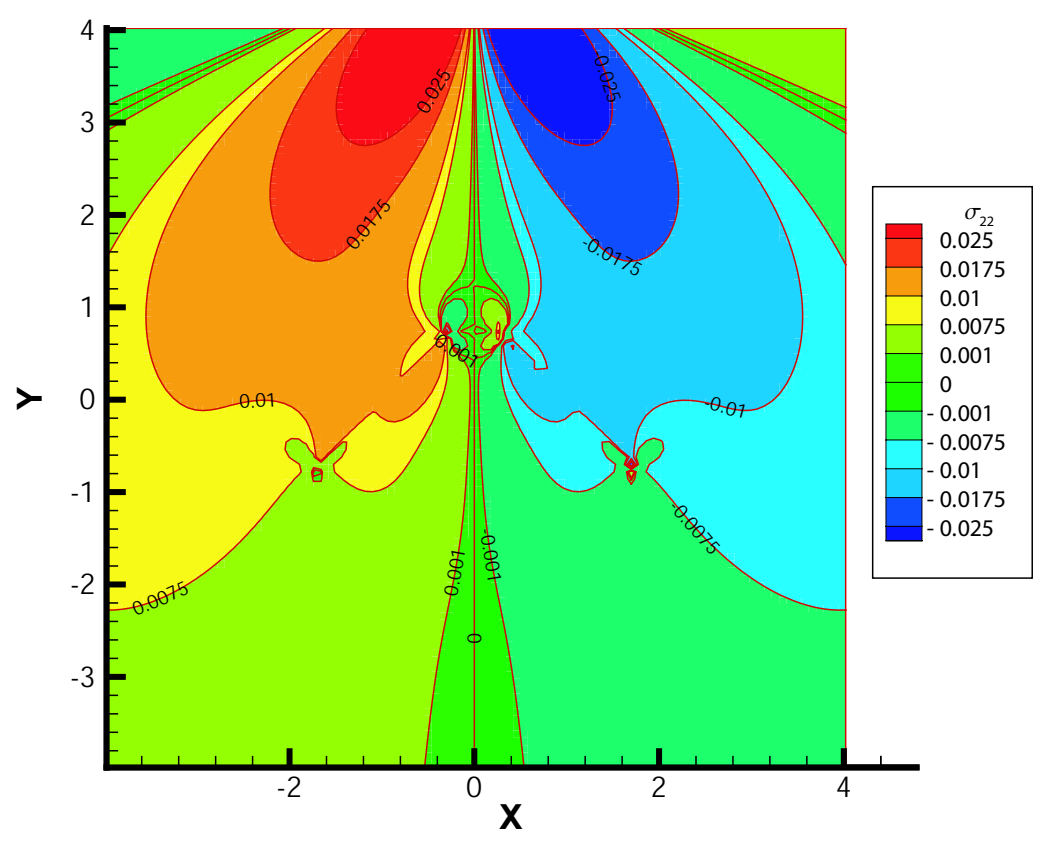

(a)

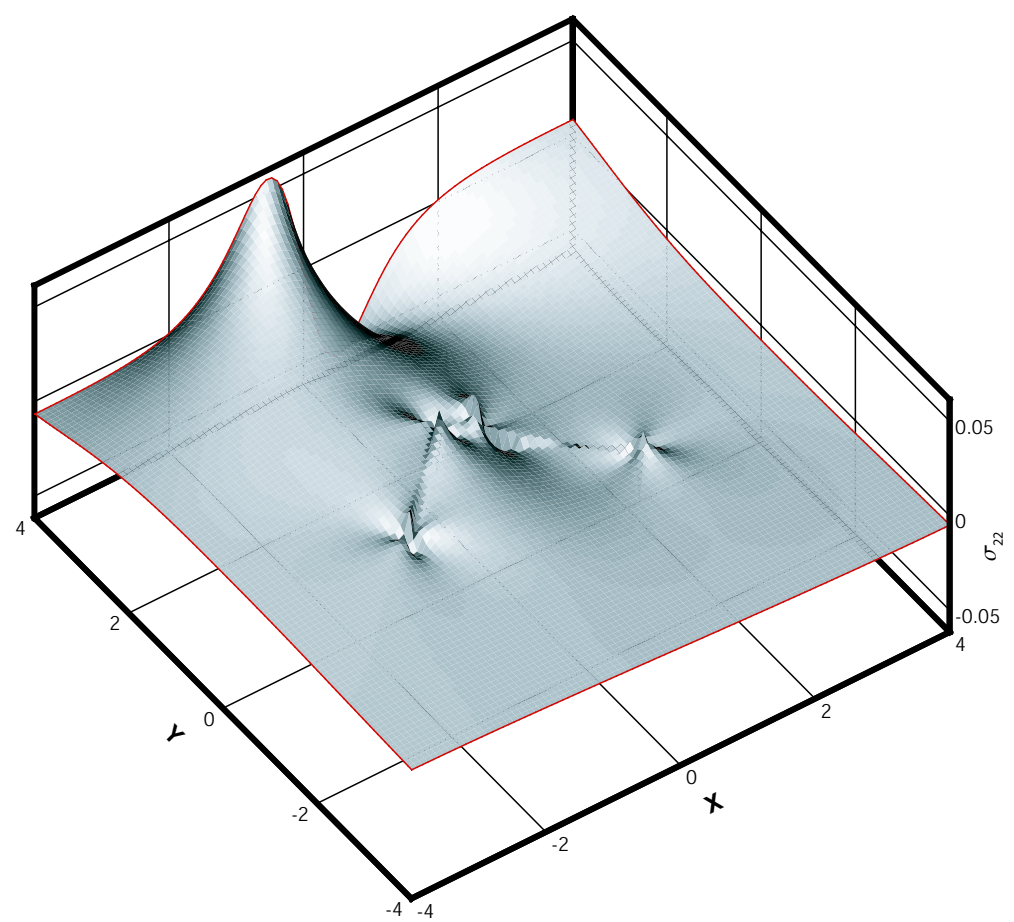

(b)

Figure 27: Variation of $\sigma_{y y}$ by $f_{x}=1$ as a function of location in infinite region with two inclined cracks for (a) 2-D color contour and (b) 3-D shaded plot. 


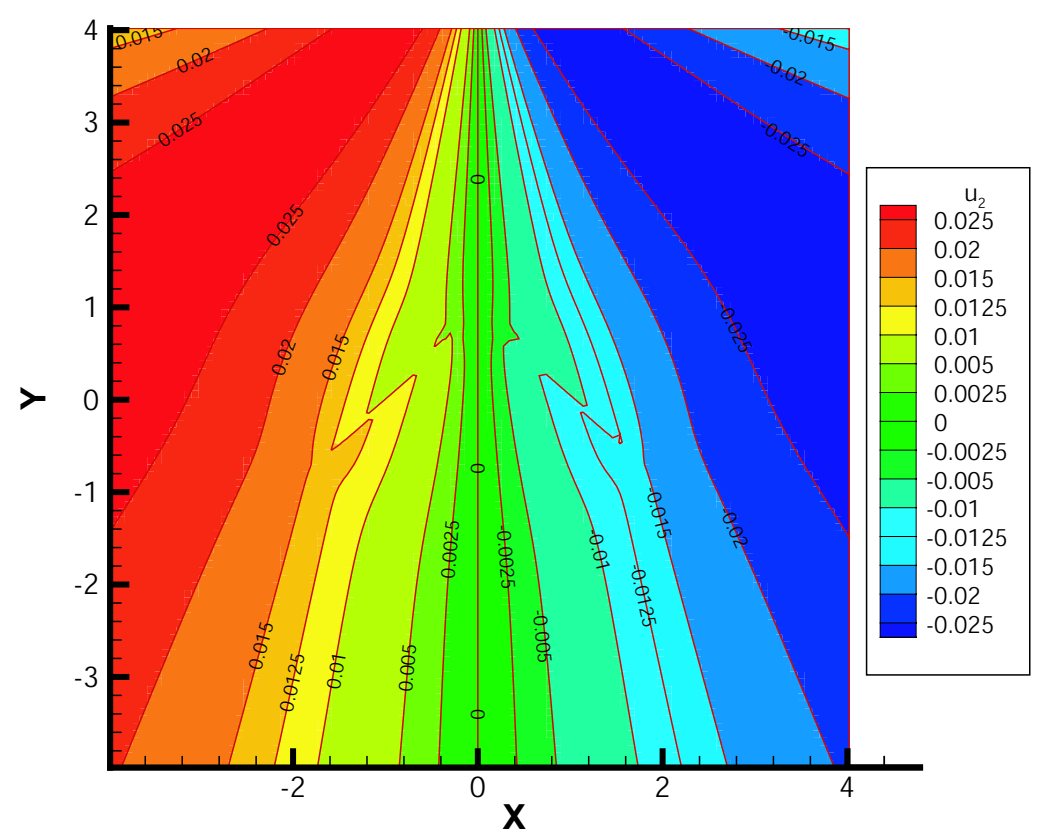

(a)

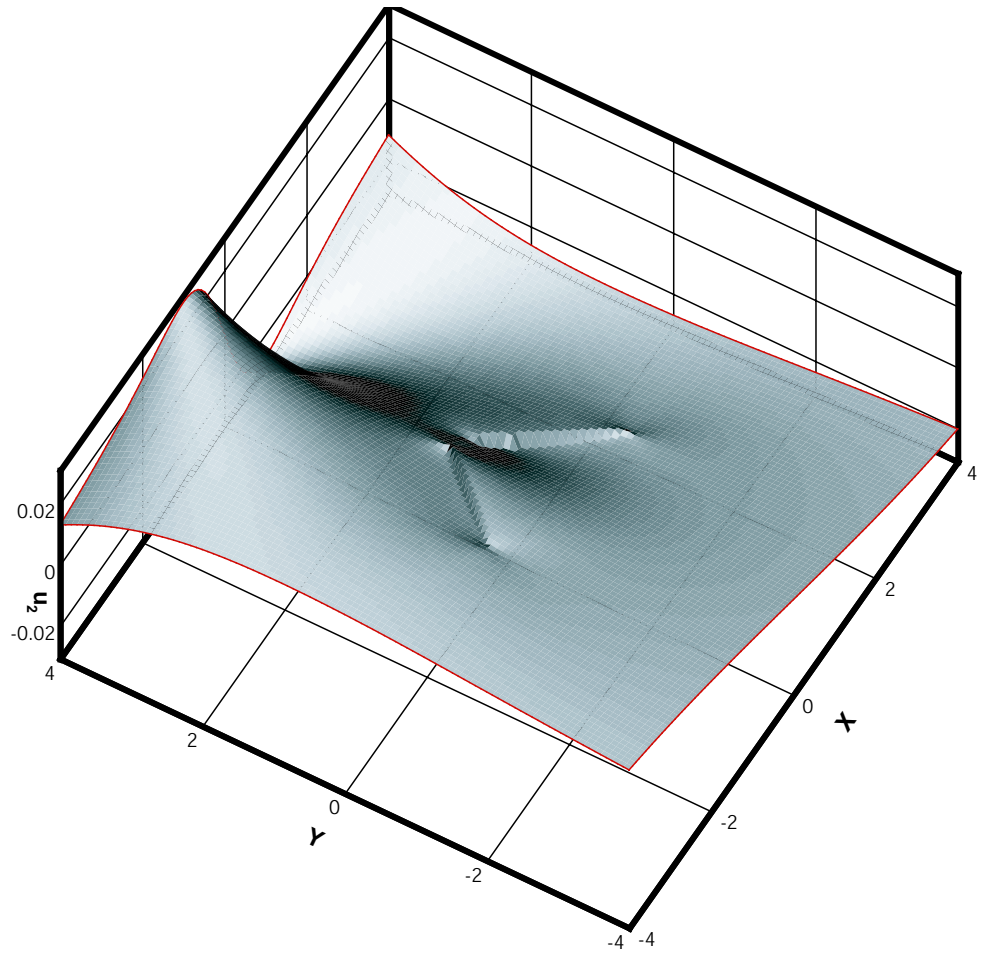

(b)

Figure 28: Variation of $u_{y}$ by $f_{x}=1$ as a function of location in infinite region with two inclined cracks for (a) 2-D color contour and (b) 3-D shaded plot. 


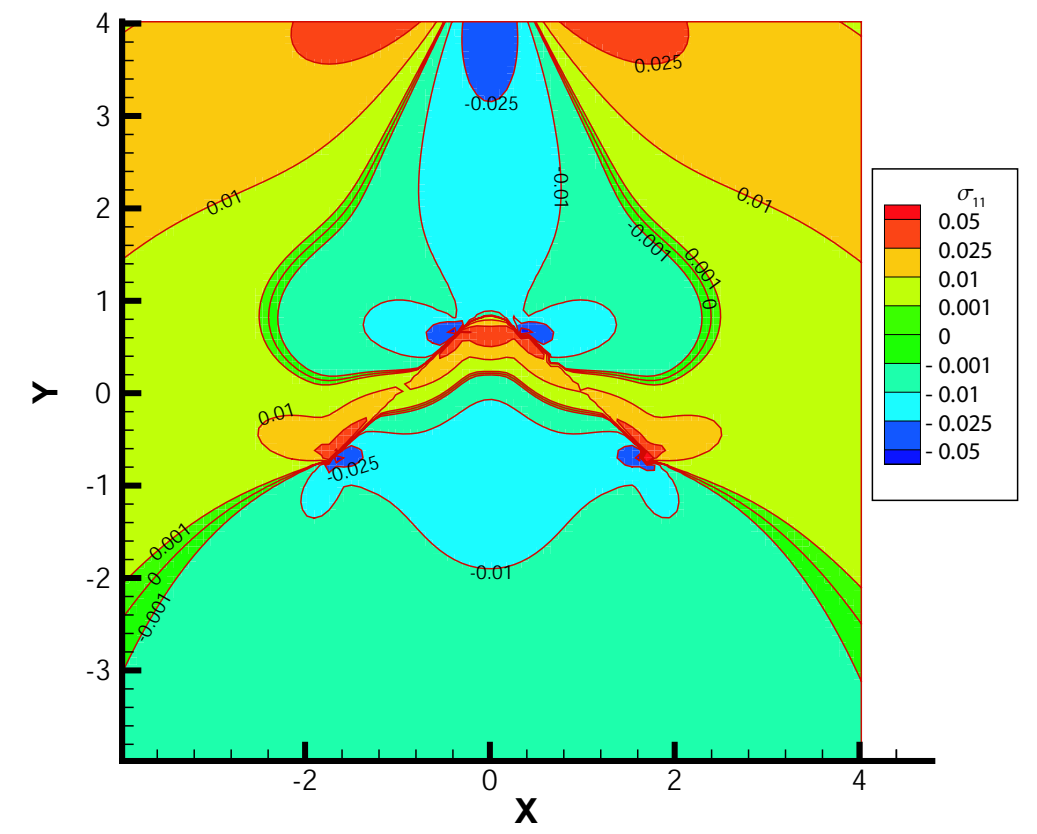

(a)

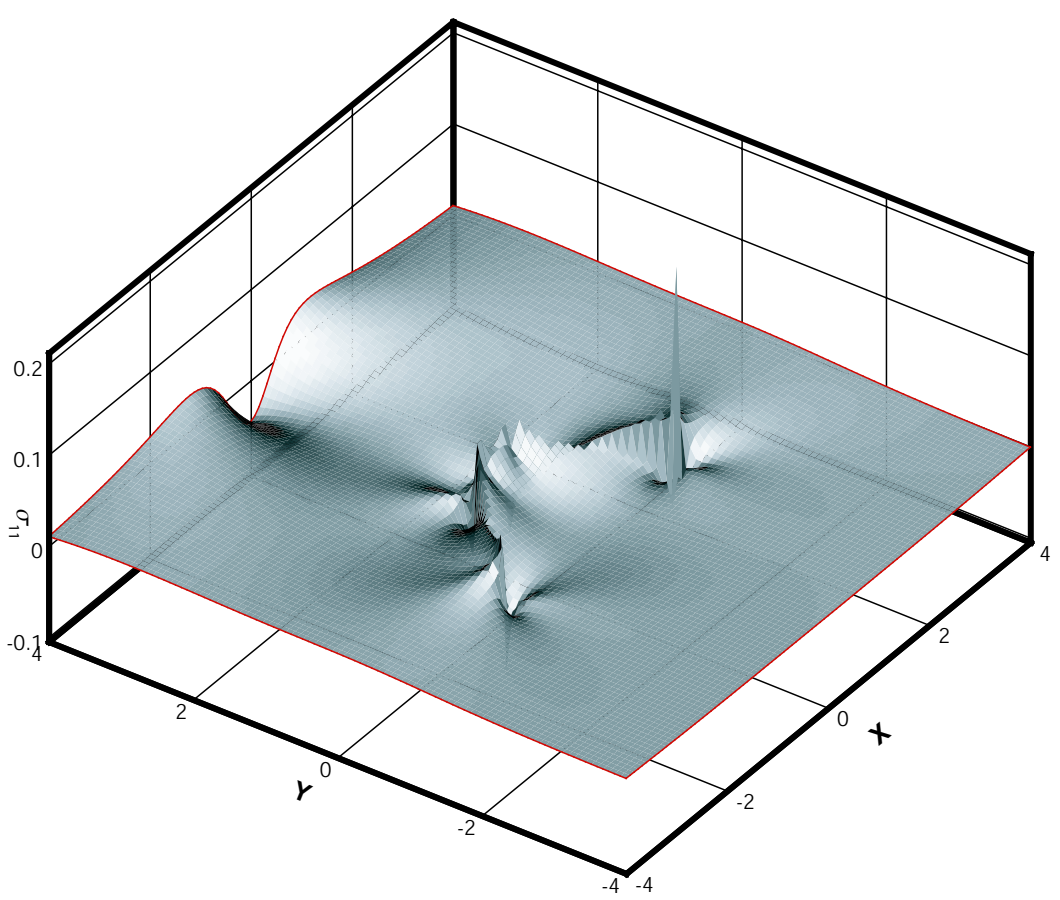

(b)

Figure 29: Variation of $\sigma_{x x}$ by $f_{y}=1$ as a function of location in infinite region with two inclined cracks for (a) 2-D color contour and (b) 3-D shaded plot. 